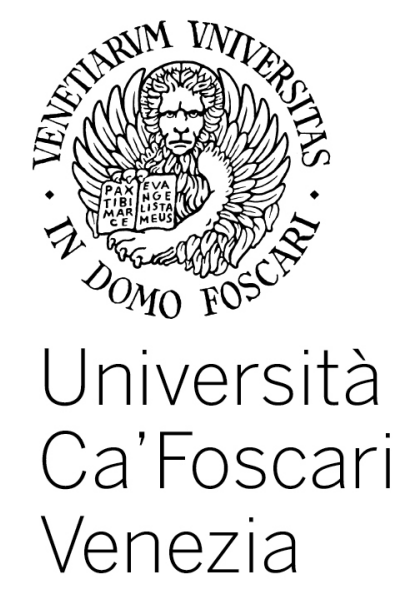

Department
of Management

Working Paper Series

I. Bykadorov, A. Ellero, S. Funari,

S. Kokovin and P. Molchanov

Pro-competitive effects and harmful trade liberalization in multi-country world

Working Paper n. 6/2015 August 2015

ISSN: 2239-2734 
This Working Paper is published under the auspices of the Department of Management at Università $\mathrm{Ca}^{\prime}$ Foscari Venezia. Opinions expressed herein are those of the authors and not those of the Department or the University. The Working Paper series is designed to divulge preliminary or incomplete work, circulated to favour discussion and comments. Citation of this paper should consider its provisional nature. 


\title{
Pro-competitive effects and harmful trade liberalization in multi-country world*
}

\author{
IGOR BYKADOROV \\ <bykadorov.igor@mail.ru> \\ Department of Management \\ Ca' Foscari University of Venice \\ Sobolev Institute of Mathematics SB RAS \\ MSSE NRU Higher School of Economics \\ NRU Novosibirsk State University \\ Stefania Funari \\ $<$ funari@unive.it> \\ Department of Management \\ Ca' Foscari University of Venice \\ PaVel Molchanov \\ $<$ MrMPS1992@gmail.com> \\ MSSE NRU Higher School of Economics
}

\author{
ANDREA Ellero \\ $<$ ellero@unive.it> \\ Department of Management \\ Ca' Foscari University of Venice
}

(July 2015)

\begin{abstract}
We study a multi-country trade model with two types of countries (big and small ones). The model generalizes the case of two countries analyzed in [3] and wonder whether there could be harm from trade. Monopolistic competition, iceberg type trade costs and variable elasticity of substitution (VES) are among the model assumptions. The effects of trade liberalization on price, production and welfare are analyzed, in cases of free trade and autarky.
\end{abstract}

Keywords: Monopolistic competition trade model, variable elasticity of substitution, iceberg type trade costs, free trade, autarky.

JEL Classification Numbers: F12, L13, D43.

\footnotetext{
${ }^{*}$ We are indebted to Evgeny Zhelobodko who started this study, to Jacques-Francois Thisse for encouragement, Federico Etro, Sergey Kichko, Yasusada Murata, Federicof Trionfetti, Philip Ushchev, and Dao-Zhi Zeng for valuable comments. Also we acknowledge seminar participants at MSSE NRU Higher School of Economics and at Department of Management Ca' Foscari University of Venice. The research project is partially financed by the Department of Management of Ca' Foscari University of Venice (grant decree No 571/2014, for SSD SECS-S/06), by the Russian government (grant No 11.G34.31.0059) and by RFBR (grant No 15-06-05666).
} 


\section{Correspondence to:}

Igor Bykadorov Department of Management

Ca' Foscari University of Venice

E-mail: $\quad$ igor.bykadorov@unive.it

bykadorov.igor@mail.ru 


\section{Introduction}

Welfare gains from trade in monopolistic competition is an important issue in both theoretical and empirical research in international trade (see [7]).

The commonly called pro-competitive effects, based on the idea that international trade increases competition, may influence gains from trade, as observed in [1] and [2]; however, the intensity of these effects on welfare is not taken for granted. Arkolakis et al. [2] found that, under variable elasticity of substitution (VES) that generates variable markups, gains from trade liberalization are slightly lower than than those occurred in models with CES utility; therefore pro-competitive effects are "elusive" in this case.

Even losses from trade are proved under VES conditions; indeed, Bykadorov et al. [4] established necessary and sufficient conditions for welfare reduction in a generalized DixitStiglitz-Krugman model (see [5], [6]) when a general-form (unspecified) additive utility and non-linear costs are assumed. The conditions under which losses may occur when jumping from autarky to free trade are related to misaligned preferences and specific costs. Therefore, these conditions are so stringent that [4] concludes that trade losses are unlikely in the real economy.

This paper, in fact, generalizes the Krugman's trade model in monopolistic competition with two asymmetric countries presented in [3] and wonders whether there could be losses from trade liberalization in a multi-country framework.

In case of symmetric countries, [3] proves that under VES there is not monotonicity of gains from trade when trade freeness rises; indeed, the first step into trade is proved to be harmful, while the highest gains happen near free trade. In case of two asymmetric countries, Home (bigger) and Foreign (smaller), [3] found similar results in terms of losses from trade, even though the bigger country showed higher wages and higher welfare than the smaller one. Should such non-monotonicity hold in a multi-country world?

We consider an economy with one diversified sector with homogeneous firms, one production factor and unspecified additive utilities, with several countries divided in two groups (big and small countries): the consumer population is higher in the countries labeled as big. Besides the domestic consumption, the model assumes that trade may occur both within the countries of the same group and also among countries belonging to different groups.

Within each group of big and small countries firms are supposed to be identical, producing "varieties" of goods, one variety per firm; consumers are also identical. Moreover, we assume that labor is the only scarce production factor, all entities (consumption, production, size of firms) are measured in labor and trade is also balanced (as in [6], [3]), utilities are additive functions and trade costs $(\tau)$ are of iceberg type (as in [3]).

The adoption of iceberg trade costs, which are very common in models of international trade since Samuelson's work, allows to relate the possible trade conditions (from autarky to free trade trough trade liberalizations) to different values of the parameter $\tau$; liberalization is thus associated to a reduction of $\tau$. We distinguish a concept of partial autarky, where only some forms of trade stop, from a concept of total autarky, where all international trades stop, and we analyze the different types of autarky occurring when trade cost varies.

Under unspecified decreasingly-elastic utilities showing increasingly-elastic demand, we prove that each price decreases with trade liberalization. With big trade costs, approaching 
autarky, liberalization pushes up variety but, necessarily, welfare in each country goes down independently from the kind of trade that starts first! The reason is that the mass of firms is excessive under decreasingly-elastic utilities, whereas liberalization aggravates this market distortion with tiny compensation from increasing import. At the opposite extreme of trade cost evolution, near free trade, we find that the total output of each firm decreases and welfare increases in each country with liberalization, in spite of a decreasing wage differential. The welfare differential behave similarly, it shows big-country advantage fading through liberalization that explains fading agglomeration forces in a simple fashion. Such VES effects generalize those under CES, whereas the effects near autarky are new since CES excludes autarky (excludes zeros in the export matrix). Theoretically, our paper supports [2] also in the case of a multi-country economy: VES economy can bring smaller trade gains than CES one, it can even bring harmful trade. Practically, it warns: Do not liberalize trade gradually!

The remainder of the paper is organized as follows.

Section 2 develops the model of monopolistic competition in case of many big and small countries, by building demand and profit functions (sec. 2.1-2.2) for each group of big and small countries. Labor and trade balance conditions and welfare function are also obtained (sec. 2.3-2.4). Section 3 presents the equilibrium conditions in the symmetric case and illustrates the behavior of the equilibrium variables as trade costs increase (sec. 3.1-3.2); free trade and autarky are investigated more in detail and two propositions present the main results (sec. 3.3-3.4). A simulation is illustrated in case of a simplified situation of three big and three small countries and a suitable utility function (sec. 3.5). Section 4 presents overall conclusions. Finally, section 5 (Appendix) collects all the detailed proofs.

\section{An economy of many big and small countries}

Let us consider $K+1$ "big" identical countries and $k+1$ "small" identical countries. Big countries differ from small countries in the number of consumers, i.e., the quantity of labor. More precisely, let be

- $L$ the number of consumers (quantity of labor) in each big country,

- $l$ the number of consumers (quantity of labor) in each small country,

with $l \leq L$; so $\Gamma=L \cdot(K+1)+l \cdot(k+1)$ is the total number of consumers in all the countries. The number of firms in each country are assumed to be

- $N$ the number (or mass) of firms in each big country,

- $n$ the number (or mass) of firms in each small country.

For what concerns consumption/production, consider

- $X_{i}$ the domestic consumption of the $i$-th firm in a big country, $i \in[0, N]$,

- $Y_{i}$ the foreign consumption of the $i$-th firm in a big country due to trade with other big countries $i \in[0, N]$, 
- $z_{i}$ the foreign consumption of the $i$-th firm in a big country due to trade with small countries, $i \in[0, N]$,

- $x_{i}$ the domestic consumption of the $i$-th firm in a small country, $i \in[0, n]$,

- $y_{i}$ the foreign consumption of the $i$-th firm in a small country due to trade with other small countries, $i \in[0, n]$,

- $Z_{i}$ the foreign consumption of the $i$-th firm in a small country due to trade with big countries, $i \in[0, n]$;

and the corresponding prices

- $P_{i}^{X}, P_{i}^{Y}, P_{i}^{Z}, p_{i}^{x}, p_{i}^{y}, p_{i}^{z}$. Figure 1 illustrates the notation in the case of three big and three small countries.

Wages are assumed to be different in big and small countries:

- $w^{B}=w$ the wage in each big country,

- $w^{s} \equiv 1$ the (normalized) wage in each small country.

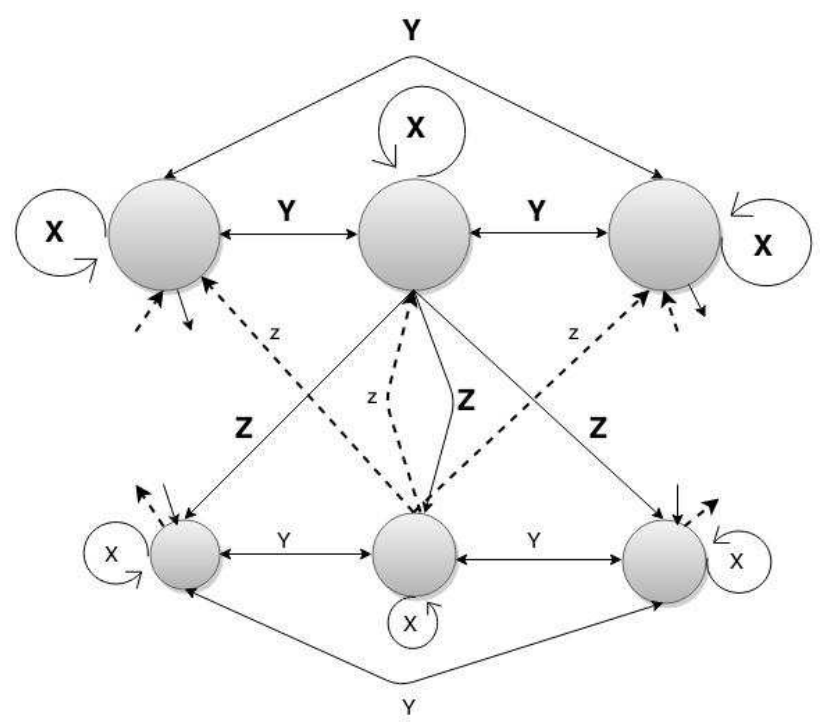

Figure 1: Graphical representation of the case of three big and three small countries

We observe that [3] studies two special cases of the outlined economy: 1) one big and one small country (i.e., $K=k=0$, the vertical case, see figure $2(\mathrm{a})$ ) and 2) $K+1$ identical countries (see figure 2(b), with $K=2$ ). 


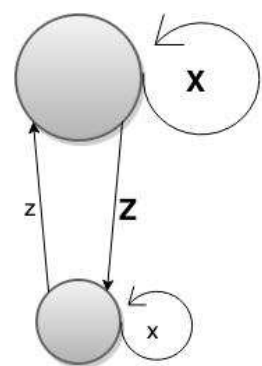

(a)

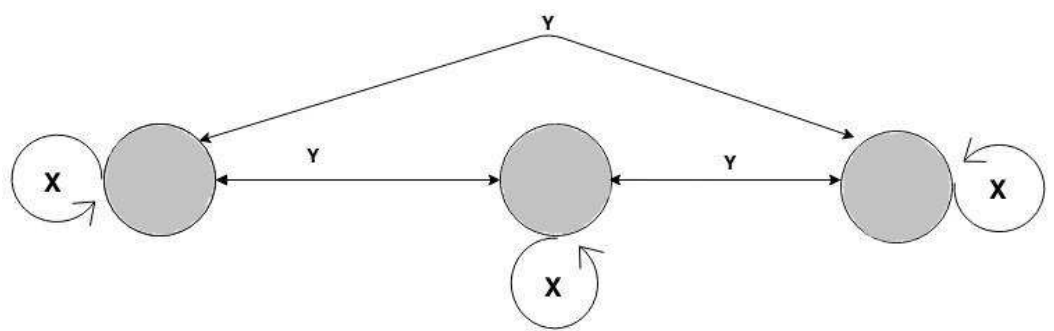

(b)

Figure 2: Graphical representation of the case of two non-identical countries (a) and three identical countries (b).

\subsection{Consumers and demand functions}

Each consumer has an elementary utility function $u(\cdot)$, three times differentiable, which satisfies (see [8])

$$
u(0)=0, u^{\prime}(\xi)>0, u^{\prime \prime}(\xi)<0,
$$

i.e., it is everywhere strictly increasing and strictly concave.

The problem of a representative consumer in a big country is given by

$$
\int_{i \in[0, N]} u\left(X_{i}\right) d i+K \cdot \int_{i \in[0, N]} u\left(Y_{i}\right) d i+(k+1) \cdot \int_{i \in[0, n]} u\left(z_{i}\right) d i \rightarrow \max ,
$$

subject to:

$$
\int_{i \in[0, N]} P_{i}^{X} X_{i} d i+K \cdot \int_{i \in[0, N]} P_{i}^{Y} Y_{i} d i+(k+1) \cdot \int_{i \in[0, n]} p_{i}^{z} z_{i} d i \leqslant w,
$$

while the problem of a representative consumer in a small country is

$$
\int_{i \in[0, n]} u\left(x_{i}\right) d i+k \cdot \int_{i \in[0, n]} u\left(y_{i}\right) d i+(K+1) \cdot \int_{i \in[0, N]} u\left(Z_{i}\right) d i \rightarrow \max ,
$$

subject to:

$$
\int_{i \in[0, n]} p_{i}^{x} x_{i} d i+k \cdot \int_{i \in[0, n]} p_{i}^{y} y_{i} d i+(K+1) \cdot \int_{i \in[0, N]} P_{i}^{Z} Z_{i} d i \leqslant 1 .
$$

From the consumer's First Order Conditions (FOC), one has the (inverse) demand functions

$$
P_{i}^{X}=\frac{u^{\prime}\left(X_{i}\right)}{\Lambda}, \quad P_{i}^{Y}=\frac{u^{\prime}\left(Y_{i}\right)}{\Lambda}, \quad p_{i}^{z}=\frac{u^{\prime}\left(z_{i}\right)}{\Lambda},
$$




$$
p_{i}^{x}=\frac{u^{\prime}\left(x_{i}\right)}{\lambda}, \quad p_{i}^{y}=\frac{u^{\prime}\left(y_{i}\right)}{\lambda}, \quad P_{i}^{Z}=\frac{u^{\prime}\left(Z_{i}\right)}{\lambda} .
$$

where $\Lambda$ and $\lambda$ are the Lagrange multipliers of the two above defined problems.

Assuming symmetry, prices can be rewritten omitting index $i$ as

$$
\begin{gathered}
P^{X}=\frac{u^{\prime}(X)}{\Lambda}, \quad P^{Y}=\frac{u^{\prime}(Y)}{\Lambda}, \quad p^{z}=\frac{u^{\prime}(z)}{\Lambda} \\
p^{x}=\frac{u^{\prime}(x)}{\lambda}, \quad p^{y}=\frac{u^{\prime}(y)}{\lambda}, \quad P^{Z}=\frac{u^{\prime}(Z)}{\lambda} .
\end{gathered}
$$

\subsection{Producers and profit functions}

Let $\tau \geq 1$ be the trade cost assumed to be of iceberg type. We remind that the basic idea behind iceberg costs is that the cost to transport a given quantity of good is paid with a portion of that quantity.

The output (the size) of the $i-t h$ firm in a big country is given by

$$
Q_{i}=L \cdot X_{i}+K \cdot \tau \cdot L \cdot Y_{i}+(k+1) \cdot \tau \cdot l \cdot Z_{i}
$$

while

$$
q_{i}=l \cdot x_{i}+k \cdot \tau \cdot l \cdot y_{i}+(K+1) \cdot \tau \cdot L \cdot z_{i}
$$

is the output (the size) of the $i-t h$ firm in a small country.

Assuming symmetry the index $i$ can be omitted allowing to rewrite outputs as

$$
Q=L \cdot X+K \cdot \tau \cdot L \cdot Y+(k+1) \cdot \tau \cdot l \cdot Z,
$$

and

$$
q=l \cdot x+k \cdot \tau \cdot l \cdot y+(K+1) \cdot \tau \cdot L \cdot z,
$$

Considering linear costs, let be

$$
C\left(Q_{i}\right)=c \cdot Q_{i}+F
$$

the total cost of the $i-t h$ firm in a big country, and

$$
C\left(q_{i}\right)=c \cdot q_{i}+F
$$

the total cost of the $i-t h$ firm in a small country. Again, assuming symmetry it is possible to omit the index $i$.

Summarizing, using the "normalized" revenue $R(\xi)=u^{\prime}(\xi) \cdot \xi$ and the demand functions, assuming symmetry, the profit $\Pi$ of a firm in a big country can be written as

$$
\Pi=L \cdot \frac{R(X)}{\Lambda}+K \cdot L \cdot \frac{R(Y)}{\Lambda}+(k+1) \cdot l \cdot \frac{R(Z)}{\lambda}-w \cdot C(Q),
$$

and the profit $\pi$ in a small country as

$$
\pi=l \cdot \frac{R(x)}{\lambda}+k \cdot l \cdot \frac{R(y)}{\lambda}+(K+1) \cdot L \cdot \frac{R(z)}{\Lambda}-C(q) .
$$




\subsection{Labor and trade balances}

Labor balances in the two kind of countries can be written as

$$
N \cdot C(Q)=L, \quad n \cdot C(q)=l .
$$

Trade balances in big and small countries are, respectively

$$
N \cdot\left(K \cdot L \cdot \frac{R(Y)}{\Lambda}+(k+1) \cdot l \cdot \frac{R(Z)}{\lambda}\right)=N \cdot K \cdot L \cdot \frac{R(Y)}{\Lambda}+n \cdot(k+1) \cdot L \cdot \frac{R(z)}{\Lambda}
$$

and

$$
n \cdot\left(k \cdot l \cdot \frac{R(y)}{\lambda}+(K+1) \cdot L \cdot \frac{R(z)}{\Lambda}\right)=n \cdot k \cdot l \cdot \frac{R(y)}{\lambda}+N \cdot(K+1) \cdot l \cdot \frac{R(Z)}{\Lambda} .
$$

Note that, after some cancellation, the two trade balances can both be rewritten by means of only one equation

$$
N \cdot l \cdot \frac{R(Z)}{\lambda}=n \cdot L \cdot \frac{R(z)}{\Lambda},
$$

i.e., by substituting $N$ and $n$ from the labor balances (3),

$$
\frac{R(Z)}{\lambda \cdot C(Q)}=\frac{R(z)}{\Lambda \cdot C(q)}
$$

which we rewrite as

$$
T B=\frac{R(Z)}{\lambda \cdot C(Q)}-\frac{R(z)}{\Lambda \cdot C(q)}=0
$$

\subsection{Welfare functions}

The welfare in each big country is

$$
W^{B}=L \cdot(N \cdot(u(X)+K \cdot u(Y))+n \cdot(k+1) \cdot u(z))
$$

while in each small country it is

$$
W^{s}=l \cdot(n \cdot(u(x)+k \cdot u(y))+N \cdot(K+1) \cdot u(Z)) .
$$

Using labor balance (3), the welfare functions can be rewritten as, respectively,

$$
W^{B}=L \cdot\left(L \cdot \frac{u(X)+K \cdot u(Y)}{C(Q)}+l \cdot(k+1) \cdot \frac{u(z)}{C(q)}\right)
$$

and

$$
W^{s}=l \cdot\left(l \cdot \frac{u(x)+k \cdot u(y)}{C(q)}+L \cdot(K+1) \cdot \frac{u(Z)}{C(Q)}\right) .
$$

The total welfare is therefore

$$
\begin{gathered}
W \equiv(K+1) \cdot W^{B}+(k+1) \cdot W^{s}= \\
=L \cdot(K+1) \cdot \frac{L \cdot u(X)+K \cdot L \cdot u(Y)+(K+1) \cdot l \cdot u(Z)}{C(Q)}+ \\
+l \cdot(k+1) \cdot \frac{l \cdot u(x)+k \cdot l \cdot u(y)+(k+1) \cdot L \cdot u(z)}{C(q)} .
\end{gathered}
$$




\section{Equilibrium}

In the monopolistic competition framework, firms are assumed to freely enter the market while their profit remains positive, which implies zero-profit (free-entry) conditions in equilibrium. In the symmetric case we get

$$
\Pi=0, \quad \pi=0 .
$$

Considering producer's First Order Conditions (FOC) in the symmetric case we obtain

$$
\frac{\partial \Pi}{\partial X}=0, \quad \frac{\partial \Pi}{\partial Y}=0, \quad \frac{\partial \Pi}{\partial Z}=0, \quad \frac{\partial \pi}{\partial x}=0, \quad \frac{\partial \pi}{\partial y}=0, \quad \frac{\partial \pi}{\partial z}=0,
$$

Second Order Conditions (SOC), since costs are assumed to be linear, are given by

$$
\frac{\partial^{2} \Pi}{\partial X \partial X}<0, \quad \frac{\partial^{2} \Pi}{\partial Y \partial Y}<0, \quad \frac{\partial^{2} \Pi}{\partial Z \partial Z}<0, \quad \frac{\partial^{2} \pi}{\partial x \partial x}<0, \quad \frac{\partial^{2} \pi}{\partial y \partial y}<0, \quad \frac{\partial^{2} \pi}{\partial z \partial z}<0,
$$

which can be easily rewritten in terms of normalized revenue $R$ as

$$
R^{\prime \prime}(X)<0, \quad R^{\prime \prime}(Y)<0, \quad R^{\prime \prime}(Z)<0, \quad R^{\prime \prime}(x)<0, \quad R^{\prime \prime}(y)<0, \quad R^{\prime \prime}(z)<0 .
$$

Let us consider some more notation that will be used in the following. Given a function $f(\xi)$, define the elasticity ${ }^{1}$

$$
E_{f}(\xi)=\frac{f^{\prime}(\xi) \cdot \xi}{f(\xi)}
$$

and the Arrow-Pratt measure

$$
r_{f}(\xi)=-\frac{f^{\prime \prime}(\xi) \cdot \xi}{f^{\prime}(\xi)}=-E_{f^{\prime}}(\xi) .
$$

Therefore SOC can be written as (see [8])

$$
r_{u^{\prime}}(X)<2, r_{u^{\prime}}(Y)<2, r_{u^{\prime}}(Z)<2, r_{u^{\prime}}(x)<2, r_{u^{\prime}}(y)<2, r_{u^{\prime}}(z)<2 .
$$

\subsection{Symmetric equilibrium}

A symmetric equilibrium is a bundle

$$
\left(X^{*}, Y^{*}, Z^{*}, x^{*}, y^{*}, z^{*}, \Lambda^{*}, \lambda^{*}, w^{*}, N^{*}, n^{*}, P^{X^{*}}, P^{Y^{*}}, P^{Z^{*}}, p^{x^{*}}, p^{y^{*}}, p^{z^{*}}\right)
$$

satisfying:

1) utility maximization (1) and (2),

2) labor and trade balances (3) and (5),

3) free entry condition (6),

\footnotetext{
${ }^{1}$ Observe that $E_{u}(\xi)<1 \forall \xi>0$. Indeed $, \forall \xi>0, E_{u}(\xi)<1 \Longleftrightarrow u^{\prime}(\xi) \cdot \xi-u(\xi)<0 \quad \forall \xi>0$. Consider the function $g(\xi)=u^{\prime}(\xi) \cdot \xi-u(\xi)$. One has $g^{\prime}(\xi) \equiv u^{\prime \prime}(\xi) \cdot \xi<0 \forall \xi>0$ due to the strict concavity of $u$. But $g(0)=u(0)=0$. Hence $g(\xi)<0 \forall \xi>0$, i.e., $u^{\prime}(\xi) \cdot \xi-u(\xi)<0 \forall \xi>0$.
} 
4) profit maximization (7) and (8).

Remark that from (3) it is possible to obtain $N$ and $n$, while (1), (2) allow to deduce prices, so that it is possible to consider only variables $(X, Y, Z, x, y, z, \Lambda, \lambda, w)$, to express the symmetric equilibrium conditions by means of a reduced system of equations (5), (6), (7), i.e.,

$$
\begin{gathered}
\frac{\partial \Pi}{\partial X}=0, \quad \frac{\partial \Pi}{\partial Y}=0, \quad \frac{\partial \Pi}{\partial Z}=0, \quad \frac{\partial \pi}{\partial x}=0, \quad \frac{\partial \pi}{\partial y}=0, \quad \frac{\partial \pi}{\partial z}=0, \\
\Pi=0, \quad \pi=0, \quad T B=0 .
\end{gathered}
$$

In the following we will mainly focus on symmetric equilibrium in the case in which the utility functions $u$ have an increasing Arrow-Pratt measure $r_{u}(\cdot)$. This means that we will focus on the so-called "pro-competitive" case. The meaning of "pro-competitiveness" has been explained, e.g. in [8], where a closed economy (only one country) was considered. It turns out that, in equilibrium, the mass of firms increases w.r.t. market size while price decreases if $r_{u}(\cdot)$ increases, and price increases if $r_{u}(\cdot)$ decreases; of course, price is constant if $r_{u}(\cdot)$ is constant, i.e., in CES-case. An increasing of the mass of firms corresponds to an increase of competition. Therefore, if $r_{u}^{\prime}(\cdot)>0$ then price decreases ("pro-competitive" case) while if $r_{u}^{\prime}(\cdot)<0$ then price increases ("anti-competitive" case). In our opinion, the "pro-competitive" case is more natural.

\subsection{Comparative statics w.r.t. trade cost $\tau$}

To simplify notation, given a variable $\varphi$ its elasticity w.r.t. trade $\operatorname{cost} \tau$ will be denoted as

$$
\mathcal{E}_{\varphi}=\mathcal{E}_{\varphi / \tau}=\frac{d \varphi}{d \tau} \cdot \frac{\tau}{\varphi} .
$$

The Lemma below, characterizing the derivatives of some variables in equilibrium, will be useful to study the behavior of equilibrium variables as the trade cost $\tau$ changes. The proofs of the following Lemmas 1, 2, 3 and 4 are reported in section 4.

Lemma 1. In equilibrium

$$
\begin{gathered}
\frac{d X}{d \tau}=\frac{1}{\tau} \cdot \frac{\mathcal{E}_{\Lambda}+\mathcal{E}_{w}}{\frac{R^{\prime \prime}(X)}{R^{\prime}(X)}}, \quad \frac{d Y}{d \tau}=\frac{1}{\tau} \cdot \frac{\mathcal{E}_{\Lambda}+\mathcal{E}_{w}+1}{\frac{R^{\prime \prime}(Y)}{R^{\prime}(Y)}}, \quad \frac{d Z}{d \tau}=\frac{1}{\tau} \cdot \frac{\mathcal{E}_{\lambda}+\mathcal{E}_{w}+1}{\frac{R^{\prime \prime}(Z)}{R^{\prime}(Z)}}, \\
\frac{d x}{d \tau}=\frac{1}{\tau} \cdot \frac{\mathcal{E}_{\lambda}}{\frac{R^{\prime \prime}(x)}{R^{\prime}(x)}}, \quad \frac{d y}{d \tau}=\frac{1}{\tau} \cdot \frac{\mathcal{E}_{\lambda}+1}{\frac{R^{\prime \prime}(y)}{R^{\prime}(y)}}, \quad \frac{d z}{d \tau}=\frac{1}{\tau} \cdot \frac{\mathcal{E}_{\Lambda}+1}{\frac{R^{\prime \prime}(z)}{R^{\prime}(z)}} \\
\frac{d Q}{d \tau}=L \cdot \frac{d X}{d \tau}+K \cdot L \cdot\left(Y+\tau \cdot \frac{d Y}{d \tau}\right)+(k+1) \cdot l \cdot\left(Z+\tau \cdot \frac{d Z}{d \tau}\right) \\
\frac{d q}{d \tau}=l \cdot \frac{d x}{d \tau}+k \cdot l \cdot\left(y+\tau \cdot \frac{d y}{d \tau}\right)+(K+1) \cdot L \cdot\left(z+\tau \cdot \frac{d z}{d \tau}\right)
\end{gathered}
$$


where $\mathcal{E}_{\Lambda}, \mathcal{E}_{\lambda}, \mathcal{E}_{w}$ satisfy the linear equations

$$
\begin{gathered}
L \cdot\left(\frac{R(X)}{\Lambda}+K \cdot \frac{R(Y)}{\Lambda}\right) \cdot\left(\mathcal{E}_{\Lambda}+\mathcal{E}_{w}\right)+(k+1) \cdot l \cdot \frac{R(Z)}{\lambda} \cdot\left(\mathcal{E}_{\lambda}+\mathcal{E}_{w}\right)+ \\
\quad+\frac{R^{\prime}(Z)}{\lambda} \cdot(K \cdot L \cdot Y+(k+1) \cdot l \cdot Z)=0, \\
(K+1) \cdot L \cdot \frac{R(z)}{\Lambda} \cdot \mathcal{E}_{\Lambda}+l \cdot\left(\frac{R(x)}{\lambda}+k \cdot \frac{R(y)}{\lambda}\right) \cdot \mathcal{E}_{\lambda}+\frac{R^{\prime}(z)}{\Lambda} \cdot(k \cdot l \cdot y+(K+1) \cdot L \cdot z)=0, \\
E_{C}(Q) \cdot \mathcal{E}_{Q}-E_{C}(q) \cdot \mathcal{E}_{q}-E_{R}(Z) \cdot \mathcal{E}_{Z}+E_{R}(z) \cdot \mathcal{E}_{z / \tau}+\left(\mathcal{E}_{\lambda}-\mathcal{E}_{\Lambda}\right)=0 .
\end{gathered}
$$

Moreover,

$$
\begin{gathered}
\mathcal{E}_{P^{X}}=\frac{r_{u}(X)}{r_{R}(X)} \cdot\left(\mathcal{E}_{\Lambda}+\mathcal{E}_{w}\right)-\mathcal{E}_{\Lambda}, \mathcal{E}_{P^{Y}}=\frac{r_{u}(Y)}{r_{R}(Y)} \cdot\left(\mathcal{E}_{\Lambda}+\mathcal{E}_{w}+1\right)-\mathcal{E}_{\Lambda}, \\
\mathcal{E}_{P^{Z}}=\frac{r_{u}(Z)}{r_{R}(Z)} \cdot\left(\mathcal{E}_{\lambda}+\mathcal{E}_{w}+1\right)-\mathcal{E}_{\lambda}, \mathcal{E}_{p^{x}}=\frac{r_{u}(x)}{r_{R}(x)} \cdot \mathcal{E}_{\lambda}-\mathcal{E}_{\lambda}, \\
\mathcal{E}_{p^{y}}=\frac{r_{u}(y)}{r_{R}(y)} \cdot\left(\mathcal{E}_{\lambda}+1\right)-\mathcal{E}_{\lambda}, \mathcal{E}_{p^{z}}=\frac{r_{u}(z)}{r_{R}(z)} \cdot\left(\mathcal{E}_{\Lambda}+1\right)-\mathcal{E}_{\Lambda} .
\end{gathered}
$$

\subsection{The case of free trade}

Consider the situation of free trade, i.e., $\tau=1$. Then

$$
\begin{gathered}
X=Y=Z=x=y=z, \\
w=1, \quad \Lambda=\lambda, \\
Q=q=((K+1) \cdot L+(k+1) \cdot l) \cdot X=\Gamma \cdot X .
\end{gathered}
$$

To simplify notation, let us drop the independent variable as in $R=R(X)=R(Y), C=$ $C(Q), E_{R}=E_{R}(X)$, and so on.

The equilibrium equations become

$$
R^{\prime}=\Lambda \cdot C^{\prime}, \quad \Gamma \cdot R=\Lambda \cdot C,
$$

hence

$$
E_{C}=E_{R} \quad\left(\equiv 1-r_{u}\right)
$$

Lemma 2. In free trade,

$$
\begin{gathered}
N=\frac{L}{C} \geq \frac{l}{C}=n, \quad \mathcal{E}_{w}=\frac{L-l}{\Gamma} \cdot E_{R} \in[0,1], \\
\mathcal{E}_{\Lambda}=-\left(l \cdot(k+1) \cdot \frac{L-l}{\Gamma} \cdot \frac{r_{R}-1}{E_{R}+r_{R}}+\Gamma-l\right) \cdot \frac{E_{R}}{\Gamma},
\end{gathered}
$$




$$
\begin{gathered}
\mathcal{E}_{\lambda}=-\left(-L \cdot(K+1) \cdot \frac{L-l}{\Gamma} \cdot \frac{r_{R}-1}{E_{R}+r_{R}}+\Gamma-l\right) \cdot \frac{E_{R}}{\Gamma}<0, \\
\frac{d X}{d \tau}=-\frac{X}{r_{R}} \cdot\left(\mathcal{E}_{\Lambda}+\mathcal{E}_{w}\right), \quad \frac{d Y}{d \tau}=-\frac{X}{r_{R}} \cdot\left(\mathcal{E}_{\Lambda}+\mathcal{E}_{w}+1\right), \quad \frac{d Z}{d \tau}=-\frac{X}{r_{R}} \cdot\left(\mathcal{E}_{\lambda}+\mathcal{E}_{w}+1\right), \\
\frac{d x}{d \tau}=-\frac{X}{r_{R}} \cdot \mathcal{E}_{\lambda}>0, \quad \frac{d y}{d \tau}=-\frac{X}{r_{R}} \cdot\left(\mathcal{E}_{\lambda}+1\right), \quad \frac{d z}{d \tau}=-\frac{X}{r_{R}} \cdot\left(\mathcal{E}_{\Lambda}+1\right), \\
\frac{d Q}{d \tau}=(\Gamma-L) \cdot \frac{r_{u}^{\prime} \cdot X^{2}}{r_{R} \cdot E_{R}}, \quad \frac{d q}{d \tau}=(\Gamma-l) \cdot \frac{r_{u}^{\prime} \cdot X^{2}}{r_{R} \cdot E_{R}} .
\end{gathered}
$$

Moreover

$$
\begin{gathered}
\frac{d N}{d \tau}=-\Gamma \cdot L \cdot(\Gamma-L) \cdot \frac{r_{u}^{\prime} \cdot X^{3}}{C \cdot r_{R}}, \quad \mathcal{E}_{N}=-\Gamma \cdot(\Gamma-L) \cdot \frac{r_{u}^{\prime} \cdot X^{3}}{r_{R}}, \\
\frac{d n}{d \tau}=-\Gamma \cdot l \cdot(\Gamma-l) \cdot \frac{r_{u}^{\prime} \cdot X^{3}}{C \cdot r_{R}}, \quad \mathcal{E}_{n}=-\Gamma \cdot(\Gamma-l) \cdot \frac{r_{u}^{\prime} \cdot X^{3}}{r_{R}}, \\
\mathcal{E}_{P^{X}}=-\frac{r_{u}^{\prime} \cdot X}{r_{R} \cdot E_{R}} \cdot \mathcal{E}_{\Lambda}+\frac{r_{u}}{r_{R}} \cdot \mathcal{E}_{w}, \quad \mathcal{E}_{P^{Y}}=-\frac{r_{u}^{\prime} \cdot X}{r_{R} \cdot E_{R}} \cdot \mathcal{E}_{\Lambda}+\frac{r_{u}}{r_{R}} \cdot\left(\mathcal{E}_{w}+1\right), \\
\mathcal{E}_{P^{Z}}=-\frac{r_{u}^{\prime} \cdot X}{r_{R} \cdot E_{R}} \cdot \mathcal{E}_{\lambda}+\frac{r_{u}}{r_{R}} \cdot\left(\mathcal{E}_{w}+1\right), \quad \mathcal{E}_{p^{x}}=-\frac{r_{u}^{\prime} \cdot X}{r_{R} \cdot E_{R}} \cdot \mathcal{E}_{\lambda}, \\
\mathcal{E}_{p^{y}}=-\frac{r_{u}^{\prime} \cdot X}{r_{R} \cdot E_{R}} \cdot \mathcal{E}_{\lambda}+\frac{r_{u}}{r_{R}}, \quad \mathcal{E}_{p^{z}}=\mathcal{E}_{p^{z}}=-\frac{r_{u}^{\prime} \cdot X}{r_{R} \cdot E_{R}} \cdot \mathcal{E}_{\Lambda}+\frac{r_{u}}{r_{R}} .
\end{gathered}
$$

In the free trade case, the welfare functions in big and small countries become, respectively

$$
\begin{gathered}
W^{B}=L \cdot(N \cdot(1+K)+n \cdot(k+1)) \cdot u= \\
=L \cdot(L \cdot(1+K)+l \cdot(k+1)) \cdot \frac{u}{C}=L \cdot \Gamma \cdot \frac{u}{C},
\end{gathered}
$$

and

$$
\begin{aligned}
& W^{s}=l \cdot(n \cdot(k+1)+N \cdot(K+1)) \cdot u= \\
= & l \cdot(l \cdot(k+1)+L \cdot(1+K)) \cdot \frac{u}{C}=l \cdot \Gamma \cdot \frac{u}{C}
\end{aligned}
$$

and the total welfare becomes $W=(K+1) \cdot W^{B}+(k+1) \cdot W^{s}=\Gamma^{2} \cdot \frac{u}{C}$.

Lemma 3. In free trade,

$$
\begin{gathered}
\frac{\partial W^{B}}{\partial \tau}=-\frac{L \cdot u}{\Gamma \cdot C \cdot r_{R}} \cdot\left(A^{B}+B^{B} \cdot r_{u}^{\prime} \cdot X\right), \\
\frac{\partial W^{s}}{\partial \tau}=-\frac{l \cdot u}{\Gamma \cdot C \cdot r_{R}} \cdot\left(A^{s}+B^{s} \cdot r_{u}^{\prime} \cdot X\right),
\end{gathered}
$$


where

$$
\begin{gathered}
A^{B}=\left(\Gamma \cdot L \cdot K+l \cdot(k+1) \cdot\left(\Gamma-L+l+(L-l) \cdot r_{u}\right)\right) \cdot \frac{r_{R} \cdot E_{u}}{E_{R}+r_{R}}>0, \\
B^{B}=(L \cdot(K+1) \cdot(\Gamma-L)+l \cdot(k+1) \cdot(\Gamma-l)) \cdot \frac{\left(\left(1-E_{u}\right) \cdot E_{R}+r_{u}^{\prime} \cdot X\right)}{\left(E_{R}+r_{R}\right) \cdot E_{R}}, \\
A^{s}=\mathcal{E}_{u} \cdot\left(L \cdot(K+1) \cdot(L-l) \cdot \frac{r_{R} \cdot E_{R}}{E_{R}+r_{R}}+\Gamma \cdot(\Gamma-l) \cdot r_{u}\right)>0, \\
B^{s}=L \cdot(K+1) \cdot(L-l) \cdot \frac{E_{u}}{E_{R}+r_{R}}+(l \cdot(k+1) \cdot(\Gamma-l)+L \cdot(K+1) \cdot(\Gamma-L))>0 .
\end{gathered}
$$

From Lemma 2 and Lemma 3 we get a characterization of consumption and variety changes when the world economy departs from free trade to a costly one:

Proposition 1. In free trade, as (almost zero) trade costs increase, domestic consumption in a small country $(x)$ increases. Moreover, in the pro-competitive case $\left(r_{u}^{\prime}>0\right)$ the domestic consumption in a big country $(X)$, outputs $Q$ and $q$ and all prices increase, while the mass of firms $N$ and $n$ and welfare $W^{B}$ and $W^{s}$ decrease; moreover

$$
\frac{d x}{d \tau}>\frac{d X}{d \tau}>0, \quad \frac{d q}{d \tau}>\frac{d Q}{d \tau}>0, \quad \frac{d N}{d \tau}<\frac{d n}{d \tau}<0, \quad \mathcal{E}_{n}<\mathcal{E}_{N}<0 .
$$

\subsection{The case of total autarky}

With autarky trade stops. We distinguish here three types of ("partial") autarky:

a) Trade among big countries stops $(Y=0)$;

b) Trade among big and small countries stops $(Z=z=0)$;

c) Trade among small countries stops $(y=0)$.

Let us say the autarky is "total" if all (International) trade stops, i.e., $a$ ), $b$ ), c) hold together. Depending on the order (w.r.t. $\tau$ ) in which $a$ ), b), c) happen, we can consider six cases:

- Case $a-b-c$, i.e. $\tau_{1} \leq \tau_{2} \leq \tau_{3}$ exist such that

$$
Y\left(\tau_{1}\right)=0, \quad Z\left(\tau_{2}\right)=z\left(\tau_{2}\right)=0, \quad y\left(\tau_{3}\right)=0 .
$$

- Case $b-a-c$, i.e. $\tau_{1} \leq \tau_{2} \leq \tau_{3}$ exist such that

$$
Z\left(\tau_{1}\right)=z\left(\tau_{1}\right)=0, \quad Y\left(\tau_{2}\right)=0, \quad y\left(\tau_{3}\right)=0 .
$$


- Case $a-c-b$, i.e. $\tau_{1} \leq \tau_{2} \leq \tau_{3}$ exist such that

$$
Y\left(\tau_{1}\right)=0, \quad y\left(\tau_{2}\right)=0, \quad Z\left(\tau_{3}\right)=z\left(\tau_{3}\right)=0 .
$$

- Case $c-a-b$, i.e. $\tau_{1} \leq \tau_{2} \leq \tau_{3}$ exist such that

$$
y\left(\tau_{1}\right)=0, \quad Y\left(\tau_{2}\right)=0, \quad Z\left(\tau_{3}\right)=z\left(\tau_{3}\right)=0 .
$$

- Case $b-c-a$, i.e. $\tau_{1} \leq \tau_{2} \leq \tau_{3}$ exist such that

$$
Z\left(\tau_{1}\right)=z\left(\tau_{1}\right)=0, \quad y\left(\tau_{2}\right)=0, \quad Y\left(\tau_{3}\right)=0 .
$$

- Case $c-b-a$, i.e. $\tau_{1} \leq \tau_{2} \leq \tau_{3}$ exist such that

$$
y\left(\tau_{1}\right)=0, \quad Z\left(\tau_{2}\right)=z\left(\tau_{2}\right)=0, \quad Y\left(\tau_{3}\right)=0 .
$$

Focusing on the study of total autarky, we are interested only in the last kind of international trade that stops, so that cases $a-b-c$ and $b-a-c, a-c-b$ and $c-a-b, b-c-a$ and $c-b-a$ coincide, i.e., we consider only three different cases:

- Case I) the last trade that stops, is among small countries.

- Case II) the last trade that stops, is among big and small countries.

- Case III) the last trade that stops, is among big countries.

Obviously, in Case I), $X, P^{X}, Q, N, w, W^{B}$ are constant w.r.t. $\tau$ on $\left[\tau_{2}, \tau_{3}\right]$, levels of trade costs for which big countries are already in a state of autarky. In the same way, in Case III), $x, p^{x}, q, n, w, W^{s}$ are constant w.r.t. $\tau$ on $\left[\tau_{2}, \tau_{3}\right]$.

Lemma 4. In total autarky,

- in case I) we have

$$
\begin{gathered}
\mathcal{E}_{\lambda}=\mathcal{E}_{\Lambda}=\frac{d x}{d \tau}==\mathcal{E}_{p^{x}}=0, \quad \mathcal{E}_{p^{y}}=\frac{1}{2} \\
\frac{d y}{d \tau}=\frac{R^{\prime}(y)}{R^{\prime \prime}(y)} \cdot \frac{1}{\tau}<0, \quad \mathcal{E}_{n}=-k \cdot l \cdot \frac{c \cdot \tau^{2}}{C(q)} \cdot \frac{d y}{d \tau}>0, \quad \frac{d q}{d \tau}=k \cdot l \cdot \tau \cdot \frac{d y}{d \tau}<0 \\
\mathcal{E}_{p^{y}}=\frac{1}{2} \quad\left(\Longrightarrow 0=\mathcal{E}_{P^{X}}=\mathcal{E}_{p^{x}}<\mathcal{E}_{p^{y}}=\frac{1}{2}\right) \\
\frac{d W^{s}}{d \tau}=k \cdot l^{2} \cdot \tau \cdot \frac{u(x) \cdot E_{R}(x)}{x} \cdot \frac{E_{u}(x)-1}{C(q)} \cdot \frac{d y}{d \tau}>0
\end{gathered}
$$


- in case II) we have

$$
\begin{gathered}
\mathcal{E}_{\lambda}=\mathcal{E}_{\Lambda}+\mathcal{E}_{w}=\frac{d X}{d \tau}=\frac{d x}{d \tau}=\mathcal{E}_{p^{x}}=0, \mathcal{E}_{P^{X}}=\mathcal{E}_{w}=\frac{\frac{R^{\prime \prime}(Z)}{\lambda \cdot C(q)}-w^{2} \cdot \frac{R^{\prime \prime}(z)}{\Lambda \cdot C(Q)}}{\frac{R^{\prime \prime}(Z)}{\lambda \cdot C(q)}+w^{2} \cdot \frac{R^{\prime \prime}(z)}{\Lambda \cdot C(Q)}} \in(-1,1), \\
\frac{d Z}{d \tau}=\frac{\frac{2}{\tau} \cdot \frac{R^{\prime}(Z)}{\lambda \cdot C(q)}}{\frac{R^{\prime \prime}(Z)}{\lambda \cdot C(q)}+w^{2} \cdot \frac{R^{\prime \prime}(z)}{\Lambda \cdot C(Q)}}<0, \quad \frac{d z}{d \tau}=w \cdot \frac{C(q)}{C(Q)} \cdot \frac{d Z}{d \tau}<0 \\
\frac{d Q}{d \tau}=l \cdot(k+1) \cdot \tau \cdot \frac{d Z}{d \tau}<0, \quad \frac{d q}{d \tau}=L \cdot(K+1) \cdot \tau \cdot \frac{d z}{d \tau}<0 \\
\mathcal{E}_{P^{Z}}=\mathcal{E}_{p^{z}}=\frac{1}{2} \cdot\left(\mathcal{E}_{w}+1\right)=\frac{1}{1+w^{2} \cdot \frac{\lambda \cdot C(q) \cdot R^{\prime \prime}(z)}{\Lambda \cdot C(Q) \cdot R^{\prime \prime}(Z)}} \in(0,1) \\
\mathcal{E}_{N}=-l \cdot(k+1) \cdot \frac{c \cdot \tau^{2}}{C(Q)} \cdot \frac{d Z}{d \tau}>0, \quad \frac{\mathcal{E}_{n}=-L \cdot(K+1) \cdot \frac{c \cdot \tau^{2}}{C(q)} \cdot \frac{d z}{d \tau}>0}{d W^{B}}=L \cdot l \cdot(k+1) \cdot w \cdot \frac{R^{\prime}(z)}{C(Q)} \cdot \frac{E_{u}(X)-1}{E_{u}(X)} \cdot \frac{d Z}{d \tau}>0 \\
\frac{d W^{s}}{d \tau}=L \cdot l \cdot(K+1) \cdot \frac{1}{w} \cdot \frac{R^{\prime}(Z)}{C(q)} \cdot \frac{E_{u}(x)-1}{E_{u}(x)} \cdot \frac{d z}{d \tau}>0
\end{gathered}
$$

- in case III) we have

$$
\begin{gathered}
\mathcal{E}_{\lambda}=\mathcal{E}_{\Lambda}=\frac{d X}{d \tau}=\mathcal{E}_{P^{X}}=0, \quad \mathcal{E}_{P^{Y}}=1 \\
\frac{d Y}{d \tau}=\frac{1}{\tau} \cdot \frac{R^{\prime}(Y)}{R^{\prime \prime}(Y)}<0, \mathcal{E}_{N}=-K \cdot L \cdot \frac{c \cdot \tau^{2}}{C(Q)} \cdot \frac{d Y}{d \tau}>0, \frac{d Q}{d \tau}=K \cdot L \cdot \tau \cdot \frac{d Y}{d \tau}<0, \\
\frac{d W^{B}}{d \tau}=L^{2} \cdot K \cdot \frac{u(X) \cdot E_{R}(X)}{X} \cdot \tau \cdot \frac{E_{u}(X)-1}{C(Q)} \cdot \frac{d Y}{d \tau}>0 .
\end{gathered}
$$

From Lemma 4 we get a characterization of consumption and variety changes, when the world economy leaves prohibitively high trade costs (autarchy) for smaller ones: 
Proposition 2. Starting from total autarchy, when trade liberalization ${ }^{2}$ begins,

- in Case I): in each small country, domestic consumption $x$ and its price $p^{x}$ remain constant; foreign consumption (of the production of a small country) y and output $q$ increase while the mass of firms $n$ and welfare $W^{s}$ decrease;

- in Case II): domestic consumptions $X$ and $x$ and their prices $P^{X}$ and $p^{x}$ remain constant; foreign consumption (in big countries of the production of a small country, and vice versa) $Z$ and $z$ and outputs $Q$ and $q$ increase while both mass of firms $N, n$ and welfare $W^{B}, W^{s}$ decrease;

- in Case III): in each big country, domestic consumption $X$ and its price $P^{X}$ remain constant; foreign consumption (of the production of another big country) $Y$ and output $Q$ increase while mass of firms $N$ and welfare $W^{B}$ both decrease.

\subsection{A numerical example}

Consider the simplified case of three big countries $(K=2)$ and three small countries $(k=2)$ with $L=3$ and $l=1$ consumers. We assume variable and fixed costs to be $c=3.33$ and $F=1$, respectively. The consumer utility is assumed to be

$$
u(x, a, m)=\frac{(a+1)^{m+1}\left((a+x)^{1-m}-a^{1-m}\right)}{1-m}-a x
$$

with $a=1, m=0.25$. In this example as trade costs increase, the trade first stops among big countries, then among big and small countries and finally also among small countries (i.e., we have sub-case $a-b-c$ of case I); this way $\tau_{1} \leq \tau_{2} \leq \tau_{3}$ exist such that $Y\left(\tau_{1}\right)=0$, $Z\left(\tau_{2}\right)=z\left(\tau_{2}\right)=0$ and $y\left(\tau_{3}\right)=0$, in particular $\tau_{1} \approx 1.43, \tau_{2} \approx 1.59$ and $\tau_{3} \approx 1.79$.

The simulation results are reported in figures 3 and 4 and illustrate the behaviors of the equilibrium variables. In particular, for what concerns free trade $(\tau=1)$ and (total) autarky $(\tau \approx 1.79)$, the behavior of all variables corresponds to the statements of Propositions 1 and 2 .

The pictures display also some interesting features of the variables for intermediate values of trade costs, recalling rather similar results of the massive simulations reported in [3] for the case of only two countries. In particular, we observe that productions $Q, q$ are not monotone, both with a maximum in $] 1, \tau_{1}[$, i.e., when there is trade among all countries but not free trade. We also observe that big countries consumption of small countries production $(Z)$ is lower than small countries consumption of big countries production $(z)$ before trade among big and small countries definitively stops. For what concerns the masses of firms in big $(N)$ and small $(n)$ countries, we observe that the mass of firms in big countries is three times the mass of firms in small countries in free trade (since $L=3 l$ ), it becomes $N \approx 4 n$

\footnotetext{
${ }^{2}$ Trade liberalization means that trade cost $\tau$ decreases.
} 
in $\tau_{1}$ (when trade among big countries stops), $N \approx 2.5 n$ in $\tau_{2}$ (when also trade among big and small countries stops) and $N \approx 2 n$ in total autarky.

The welfare has only one minimum both for big and small countries. Big countries reach their minimum welfare in $\left[1, \tau_{1}\right]$, i.e. when there is trade among all countries. It is interesting to observe that welfare in small countries have minimum in $\left[\tau_{1}, \tau_{2}\right]$ when trade costs are high enough to force big countries to stop trading with other big countries.

\section{Conclusions}

We have modified Krugman's (1979) model of international trade with variable elasticity of substitution for the case of multiple asymmetric countries. When the countries include several "big" and several "small" ones, the model remains tractable enough for analytical predictions. A surprising one is social harm from gradual trade liberalization (decreasing trade costs) at its first steps, near autarchy. Welfare decreases here because consumption of new foreign varieties grows slowly but the mass of firms drops sharply, which means business destruction by foreign competition. By contrast, welfare gains at the last steps of globalization are the most essential ones among the globalization path. The moral for policy makers is: Do not liberalize gradually, the main prize is at the end. 


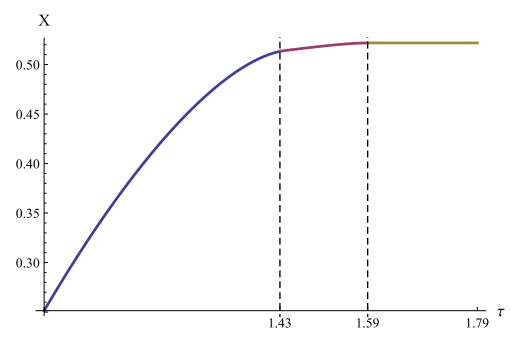

(a) $X$, big countries

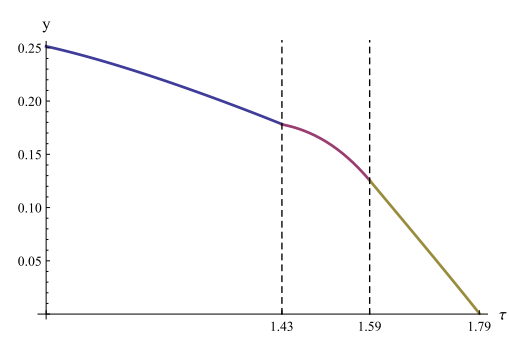

(d) $y$, small countries

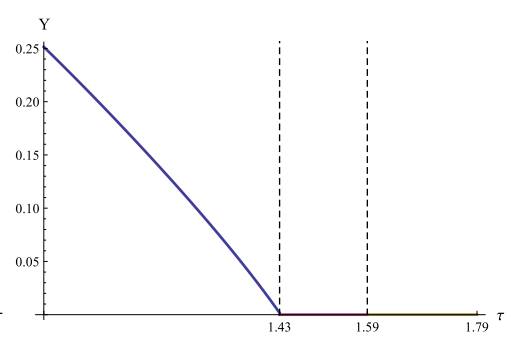

(b) $Y$, big countries

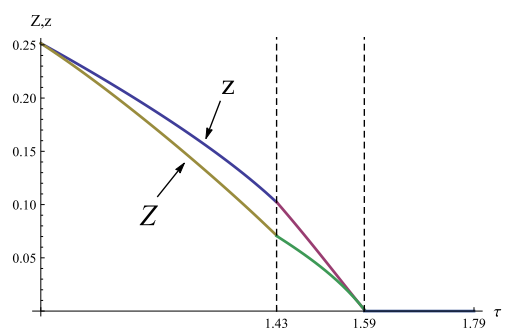

(e) $Z, z \mathrm{big} / \mathrm{small}$ countries

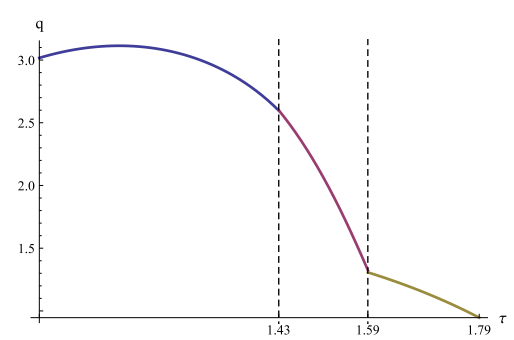

(g) q, small countries

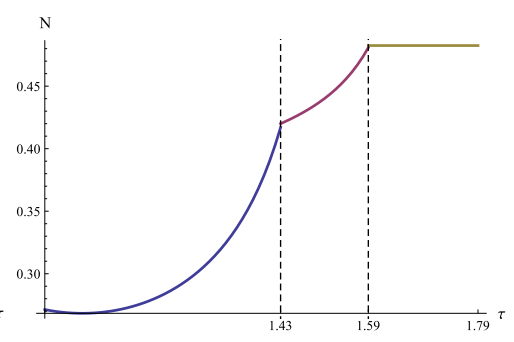

(h) $N$, big countries

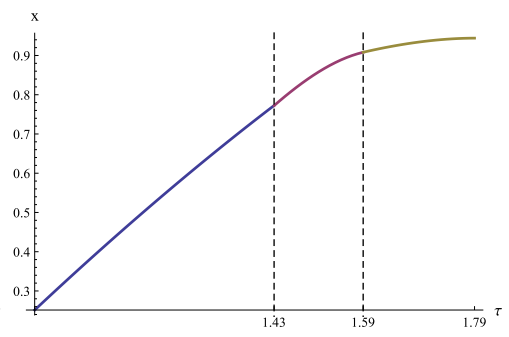

(c) $x$, small countries

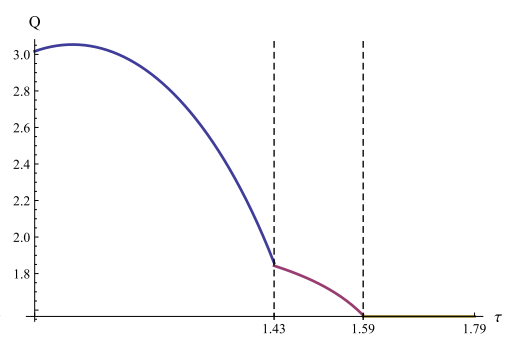

(f) $Q$, big countries

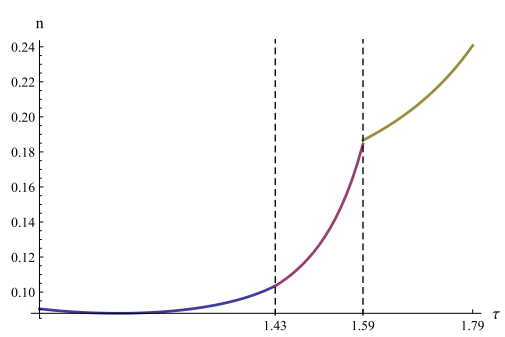

(i) $n$, small countries

Figure 3: Behavior of consumption (from (a) to (e)), output ((f), (g)) and number (mass) of firms ((h), (i)) in the example of three big and three small countries (with $L=3, l=1$, $K=2, k=2, c=3.33, F=1)$. 


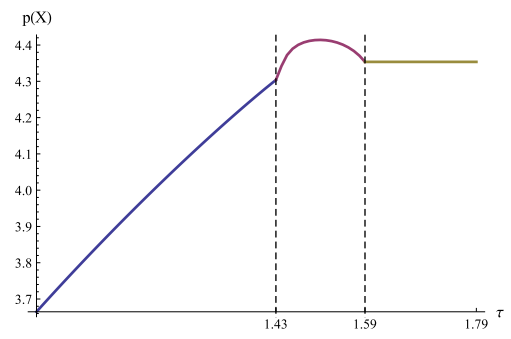

(a) $p^{X}$, big countries

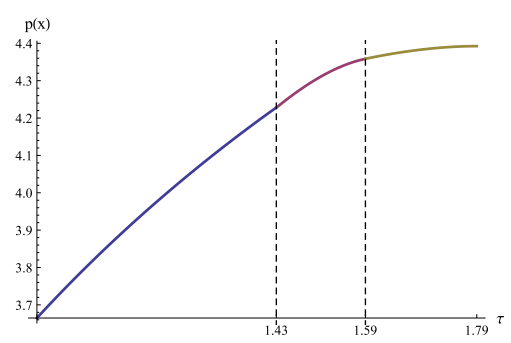

(d) $p^{x}$, small countries

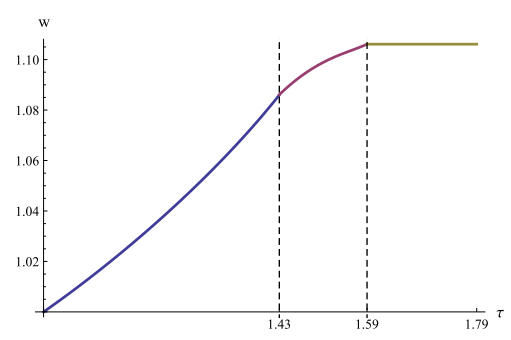

(g) $w$, big countries

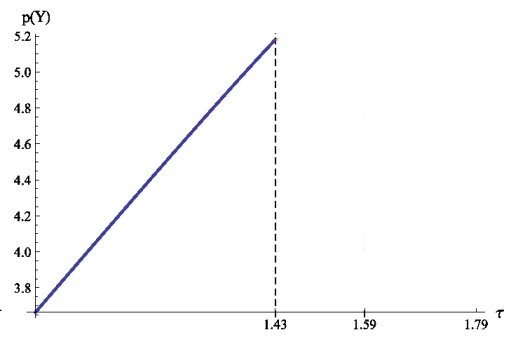

(b) $p^{Y}$, big countries

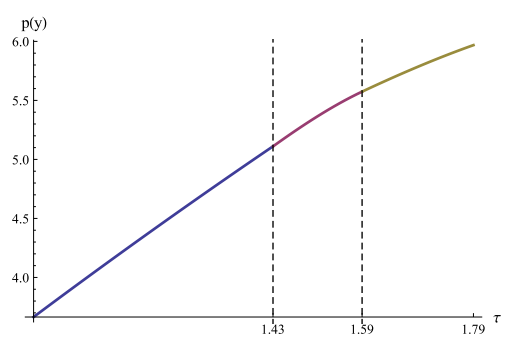

(e) $p^{y}$, small countries

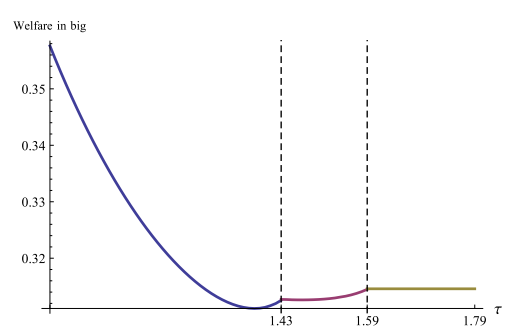

(h) welfare, big countries

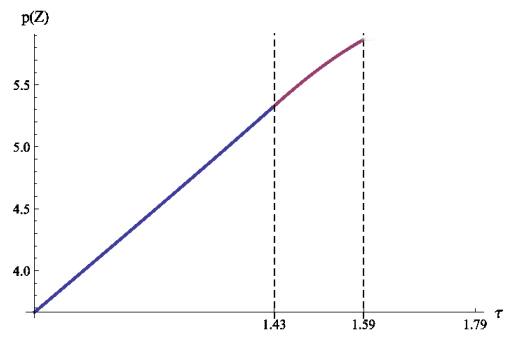

(c) $p^{Z} \mathrm{big} / \mathrm{small}$ countries

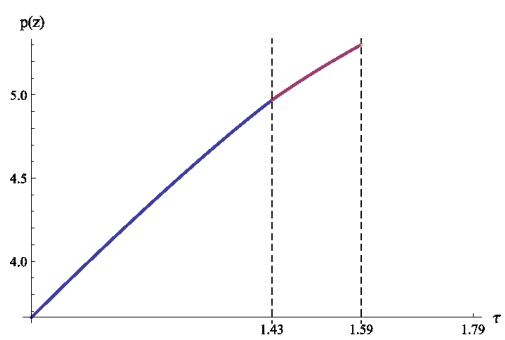

(f) $p^{z}$ small/big countries

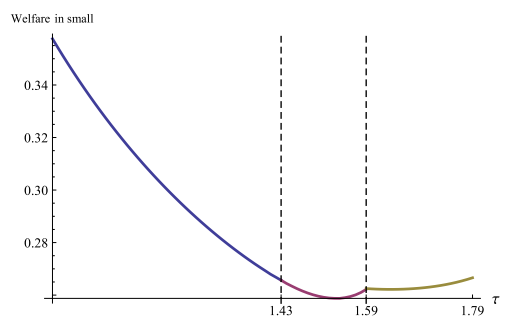

(i) welfare, small countries

Figure 4: Behavior of prices (from (a) to (f)), wage $((\mathrm{g}))$ and welfare $((\mathrm{h})$, (i)) in the example of three big and three small countries (with $L=3, l=1, K=2, k=2, c=3.33$, $F=1)$. 


\section{Appendix. Proofs}

\subsection{Proof of Lemma 1}

The study of comparative statics w.r.t. $\tau$ requires to fulfil:

$$
\begin{aligned}
& \frac{d}{d \tau}\left(\frac{\partial \Pi}{\partial X}\right)=0, \quad \frac{d}{d \tau}\left(\frac{\partial \Pi}{\partial Y}\right)=0, \quad \frac{d}{d \tau}\left(\frac{\partial \Pi}{\partial Z}\right)=0, \\
& \frac{d}{d \tau}\left(\frac{\partial \pi}{\partial x}\right)=0, \quad \frac{d}{d \tau}\left(\frac{\partial \pi}{\partial y}\right)=0, \quad \frac{d}{d \tau}\left(\frac{\partial \pi}{\partial z}\right)=0, \\
& \frac{d \Pi}{d \tau}=0, \quad \frac{d \pi}{d \tau}=0, \quad \frac{d}{d \tau}(T B)=0,
\end{aligned}
$$

where

$$
T B=\frac{R(Z)}{\lambda \cdot C(Q)}-\frac{R(z)}{\Lambda \cdot C(q)} .
$$

The above equations can be rewritten as

$$
\begin{gathered}
\frac{\partial^{2} \Pi}{\partial X \partial X} \cdot \frac{d X}{d \tau}+\frac{\partial^{2} \Pi}{\partial X \partial Y} \cdot \frac{d Y}{d \tau}+\frac{\partial^{2} \Pi}{\partial X \partial Z} \cdot \frac{d Z}{d \tau}+\frac{\partial^{2} \Pi}{\partial X \partial \Lambda} \cdot \frac{d \Lambda}{d \tau}+\frac{\partial^{2} \Pi}{\partial X \partial \lambda} \cdot \frac{d \lambda}{d \tau}+\frac{\partial^{2} \Pi}{\partial X \partial w} \cdot \frac{d w}{d \tau}+\frac{\partial^{2} \Pi}{\partial X \partial \tau}=0 \\
\frac{\partial^{2} \Pi}{\partial Y \partial X} \cdot \frac{d X}{d \tau}+\frac{\partial^{2} \Pi}{\partial Y \partial Y} \cdot \frac{d Y}{d \tau}+\frac{\partial^{2} \Pi}{\partial Y \partial Z} \cdot \frac{d Z}{d \tau}+\frac{\partial^{2} \Pi}{\partial Y \partial \Lambda} \cdot \frac{d \Lambda}{d \tau}+\frac{\partial^{2} \Pi}{\partial Y \partial \lambda} \cdot \frac{d \lambda}{d \tau}+\frac{\partial^{2} \Pi}{\partial Y \partial w} \cdot \frac{d w}{d \tau}+\frac{\partial^{2} \Pi}{\partial Y \partial \tau}=0 \\
\frac{\partial^{2} \Pi}{\partial Z \partial X} \cdot \frac{d X}{d \tau}+\frac{\partial^{2} \Pi}{\partial Z \partial Y} \cdot \frac{d Y}{d \tau}+\frac{\partial^{2} \Pi}{\partial Z \partial Z} \cdot \frac{d Z}{d \tau}+\frac{\partial^{2} \Pi}{\partial Z \partial \Lambda} \cdot \frac{d \Lambda}{d \tau}+\frac{\partial^{2} \Pi}{\partial Z \partial \lambda} \cdot \frac{d \lambda}{d \tau}+\frac{\partial^{2} \Pi}{\partial Z \partial w} \cdot \frac{d w}{d \tau}+\frac{\partial^{2} \Pi}{\partial Z \partial \tau}=0 \\
\frac{\partial^{2} \pi}{\partial x \partial x} \cdot \frac{d x}{d \tau}+\frac{\partial^{2} \pi}{\partial x \partial y} \cdot \frac{d y}{d \tau}+\frac{\partial^{2} \pi}{\partial x \partial z} \cdot \frac{d z}{d \tau}+\frac{\partial^{2} \pi}{\partial x \partial \Lambda} \cdot \frac{d \Lambda}{d \tau}+\frac{\partial^{2} \pi}{\partial x \partial \lambda} \cdot \frac{d \lambda}{d \tau}+\frac{\partial^{2} \pi}{\partial x \partial \tau}=0 \\
\frac{\partial^{2} \pi}{\partial y \partial x} \cdot \frac{d x}{d \tau}+\frac{\partial^{2} \pi}{\partial y \partial y} \cdot \frac{d y}{d \tau}+\frac{\partial^{2} \pi}{\partial y \partial z} \cdot \frac{d z}{d \tau}+\frac{\partial^{2} \pi}{\partial y \partial \Lambda} \cdot \frac{d \Lambda}{d \tau}+\frac{\partial^{2} \pi}{\partial y \partial \lambda} \cdot \frac{d \lambda}{d \tau}+\frac{\partial^{2} \pi}{\partial y \partial \tau}=0 \\
\frac{\partial^{2} \pi}{\partial z \partial x} \cdot \frac{d x}{d \tau}+\frac{\partial^{2} \pi}{\partial z \partial y} \cdot \frac{d y}{d \tau}+\frac{\partial^{2} \pi}{\partial z \partial z} \cdot \frac{d z}{d \tau}+\frac{\partial^{2} \pi}{\partial z \partial \Lambda} \cdot \frac{d \Lambda}{d \tau}+\frac{\partial^{2} \pi}{\partial z \partial \lambda} \cdot \frac{d \lambda}{d \tau}+\frac{\partial^{2} \pi}{\partial z \partial \tau}=0 \\
\frac{\partial \Pi}{\partial X} \cdot \frac{d X}{d \tau}+\frac{\partial \Pi}{\partial Y} \cdot \frac{d Y}{d \tau}+\frac{\partial \Pi}{\partial Z} \cdot \frac{d Z}{d \tau}+\frac{\partial \Pi}{\partial \Lambda} \cdot \frac{d \Lambda}{d \tau}+\frac{\partial \Pi}{\partial \lambda} \cdot \frac{d \lambda}{d \tau}+\frac{\partial \Pi}{\partial w} \cdot \frac{d w}{d \tau}+\frac{\partial \Pi}{\partial \tau}=0 \\
\frac{\partial \pi}{\partial x} \cdot \frac{d x}{d \tau}+\frac{\partial \pi}{\partial y} \cdot \frac{d y}{d \tau}+\frac{\partial \pi}{\partial z} \cdot \frac{d z}{d \tau}+\frac{\partial \pi}{\partial \Lambda} \cdot \frac{d \Lambda}{d \tau}+\frac{\partial \pi}{\partial \lambda} \cdot \frac{d \lambda}{d \tau}+\frac{\partial \pi}{\partial \tau}=0 \\
\frac{\partial T B}{\partial X} \cdot \frac{d X}{d \tau}+\frac{\partial T B}{\partial Y} \cdot \frac{d Y}{d \tau}+\frac{\partial T B}{\partial Z} \cdot \frac{d Z}{d \tau}+\frac{\partial T B}{\partial x} \cdot \frac{d x}{d \tau}+\frac{\partial T B}{\partial y} \cdot \frac{d y}{d \tau}+\frac{\partial T B}{\partial z} \cdot \frac{d z}{d \tau}+ \\
+\frac{d T B}{\partial \Lambda} \cdot \frac{d \Lambda}{d \tau}+\frac{\partial T B}{\partial \lambda} \cdot \frac{d \lambda}{d \tau}+\frac{\partial T B}{\partial \tau}=0
\end{gathered}
$$

i.e., using producer's FOC, Free Entry and linearity of Costs,

$$
\begin{gathered}
\frac{R^{\prime \prime}(X)}{\Lambda} \cdot \frac{d X}{d \tau}-\frac{R^{\prime}(X)}{\Lambda^{2}} \cdot \frac{d \Lambda}{d \tau}-C^{\prime} \cdot \frac{d w}{d \tau}=0 \\
\frac{R^{\prime \prime}(Y)}{\Lambda} \cdot \frac{d Y}{d \tau}-\frac{R^{\prime}(Y)}{\Lambda^{2}} \cdot \frac{d \Lambda}{d \tau}-\tau \cdot C^{\prime} \cdot \frac{d w}{d \tau}-w \cdot C^{\prime}=0
\end{gathered}
$$




$$
\begin{aligned}
& \frac{R^{\prime \prime}(Z)}{\lambda} \cdot \frac{d Z}{d \tau}-\frac{R^{\prime}(Z)}{\lambda^{2}} \cdot \frac{d \lambda}{d \tau}-\tau \cdot C^{\prime} \cdot \frac{d w}{d \tau}-w \cdot C^{\prime}=0 \\
& \frac{R^{\prime \prime}(x)}{\lambda} \cdot \frac{d x}{d \tau}-\frac{R^{\prime}(x)}{\lambda^{2}} \cdot \frac{d \lambda}{d \tau}=0 \\
& \frac{R^{\prime \prime}(y)}{\lambda} \cdot \frac{d y}{d \tau}-\frac{R^{\prime}(y)}{\lambda^{2}} \cdot \frac{d \lambda}{d \tau}-C^{\prime}=0 \\
& \frac{R^{\prime \prime}(z)}{\Lambda} \cdot \frac{d z}{d \tau}-\frac{R^{\prime}(z)}{\Lambda^{2}} \cdot \frac{d \Lambda}{d \tau}-C^{\prime}=0 \\
& -L \cdot\left(\frac{R(X)}{\Lambda^{2}}+K \cdot \frac{R(Y)}{\Lambda^{2}}\right) \cdot \frac{d \Lambda}{d \tau}-(k+1) \cdot l \cdot \frac{R(Z)}{\lambda^{2}} \cdot \frac{d \lambda}{d \tau}-C(Q) \cdot \frac{d w}{d \tau}-w \cdot C^{\prime} \cdot(K \cdot L \cdot Y+(k+1) \cdot l \cdot Z)=0 \\
& -(K+1) \cdot L \cdot \frac{R(z)}{\Lambda^{2}} \cdot \frac{d \Lambda}{d \tau}-l \cdot\left(\frac{R(x)}{\lambda^{2}}+k \cdot \frac{R(y)}{\lambda^{2}}\right) \cdot \frac{d \lambda}{d \tau}-C^{\prime} \cdot(k \cdot l \cdot y+(K+1) \cdot L \cdot z)=0 \\
& -\frac{R(Z) \cdot C^{\prime} \cdot L}{\lambda \cdot(C(Q))^{2}} \cdot \frac{d X}{d \tau}-\frac{R(Z) \cdot C^{\prime} \cdot K \cdot \tau \cdot L}{\lambda \cdot(C(Q))^{2}} \cdot \frac{d Y}{d \tau}+\frac{R^{\prime}(Z) \cdot C(Q)-R(Z) \cdot C^{\prime} \cdot(k+1) \cdot \tau \cdot l}{\lambda \cdot(C(Q))^{2}} \cdot \frac{d Z}{d \tau}+ \\
& +\frac{R(z) \cdot C^{\prime} \cdot l}{\Lambda \cdot(C(q))^{2}} \cdot \frac{d x}{d \tau}+\frac{R(z) \cdot C^{\prime} \cdot k \cdot \tau \cdot l}{\Lambda \cdot(C(q))^{2}} \cdot \frac{d y}{d \tau}-\frac{R^{\prime}(z) \cdot C(q)-R(z) \cdot C^{\prime} \cdot(K+1) \cdot \tau \cdot L}{\Lambda \cdot(C(q))^{2}} \cdot \frac{d z}{d \tau}+ \\
& +\frac{R(z)}{\Lambda^{2} \cdot C(q)} \cdot \frac{d \Lambda}{d \tau}-\frac{R(Z)}{\lambda^{2} \cdot C(Q)} \cdot \frac{d \lambda}{d \tau}+ \\
& +C^{\prime} \cdot\left(\frac{R(z) \cdot(k \cdot l \cdot y+(K+1) \cdot L \cdot z)}{\Lambda \cdot(C(q))^{2}}-\frac{R(Z) \cdot(K \cdot L \cdot Y+(k+1) \cdot l \cdot Z)}{\lambda \cdot(C(Q))^{2}}\right)=0
\end{aligned}
$$

i.e., using again producer's FOC, Free Entry, and elasticities w.r.t. $\tau$ for $\Lambda, \lambda, w$ :

$$
\begin{gathered}
\frac{d X}{d \tau}=\frac{1}{\tau} \cdot \frac{\mathcal{E}_{\Lambda}+\mathcal{E}_{w}}{\frac{R^{\prime \prime}(X)}{R^{\prime}(X)}}, \quad \frac{d Y}{d \tau}=\frac{1}{\tau} \cdot \frac{\mathcal{E}_{\Lambda}+\mathcal{E}_{w}+1}{\frac{R^{\prime \prime}(Y)}{R^{\prime}(Y)},} \quad \frac{d Z}{d \tau}=\frac{1}{\tau} \cdot \frac{\mathcal{E}_{\lambda}+\mathcal{E}_{w}+1}{\frac{R^{\prime \prime}(Z)}{R^{\prime}(Z)}}, \\
\frac{d x}{d \tau}=\frac{1}{\tau} \cdot \frac{\mathcal{E}_{\lambda}}{\frac{R^{\prime \prime}(x)}{R^{\prime}(x)}}, \quad \frac{d y}{d \tau}=\frac{1}{\tau} \cdot \frac{\mathcal{E}_{\lambda}+1}{\frac{R^{\prime \prime}(y)}{R^{\prime}(y)}}, \quad \frac{d z}{d \tau}=\frac{1}{\tau} \cdot \frac{\mathcal{E}_{\Lambda}+1}{\frac{R^{\prime \prime}(z)}{R^{\prime}(z)}} \\
L \cdot\left(\frac{R(X)}{\Lambda}+K \cdot \frac{R(Y)}{\Lambda}\right) \cdot\left(\mathcal{E}_{\Lambda}+\mathcal{E}_{w}\right)+(k+1) \cdot l \cdot \frac{R(Z)}{\lambda} \cdot\left(\mathcal{E}_{\lambda}+\mathcal{E}_{w}\right)+\frac{R^{\prime}(Z)}{\lambda} \cdot(K \cdot L \cdot Y+(k+1) \cdot l \cdot Z)=0 \\
(K+1) \cdot L \cdot \frac{R(z)}{\Lambda} \cdot \mathcal{E}_{\Lambda}+l \cdot\left(\frac{R(x)}{\lambda}+k \cdot \frac{R(y)}{\lambda}\right) \cdot \mathcal{E}_{\lambda}+\frac{R^{\prime}(z)}{\Lambda} \cdot(k \cdot l \cdot y+(K+1) \cdot L \cdot z)=0 \\
-\frac{R(Z) \cdot C^{\prime}}{\lambda \cdot(C(Q))^{2}} \cdot\left(L \cdot \frac{d X}{d \tau}+K \cdot \tau \cdot L \cdot \frac{d Y}{d \tau}+(k+1) \cdot \tau \cdot l \cdot \frac{d Z}{d \tau}\right)+\frac{R^{\prime}(Z)}{\lambda \cdot C(Q)} \cdot \frac{d Z}{d \tau}+ \\
+\frac{R(z) \cdot C^{\prime}}{\Lambda \cdot(C(q))^{2}} \cdot\left(l \cdot \frac{d x}{d \tau}+k \cdot \tau \cdot l \cdot \frac{d y}{d \tau}+(K+1) \cdot \tau \cdot L \cdot \frac{d z}{d \tau}\right)-\frac{R^{\prime}(z)}{\Lambda \cdot C(q)} \cdot \frac{d z}{d \tau}+ \\
+\frac{R(z)}{\Lambda \cdot C(q)} \cdot \frac{1}{\tau} \cdot \mathcal{E}_{\Lambda}-\frac{R(Z)}{\lambda \cdot C(Q)} \cdot \frac{1}{\tau} \cdot \mathcal{E}_{\lambda}+ \\
+C^{\prime} \cdot\left(\frac{R(z) \cdot(k \cdot l \cdot y+(K+1) \cdot L \cdot z)}{\Lambda \cdot(C(q))^{2}}-\frac{R(Z) \cdot(K \cdot L \cdot Y+(k+1) \cdot l \cdot Z)}{\lambda \cdot(C(Q))^{2}}\right)=0 .
\end{gathered}
$$


Moreover since

$$
\begin{gathered}
\frac{d Q}{d \tau}=L \cdot \frac{d X}{d \tau}+K \cdot L \cdot\left(Y+\tau \cdot \frac{d Y}{d \tau}\right)+(k+1) \cdot l \cdot\left(Z+\tau \cdot \frac{d Z}{d \tau}\right) \\
\frac{d q}{d \tau}=l \cdot \frac{d x}{d \tau}+k \cdot l \cdot\left(y+\tau \cdot \frac{d y}{d \tau}\right)+(K+1) \cdot L \cdot\left(z+\tau \cdot \frac{d z}{d \tau}\right)
\end{gathered}
$$

the last equation can be rewritten as

$\frac{R(Z)}{\lambda \cdot C(Q)} \cdot\left(\frac{C^{\prime}}{C(Q)} \cdot \frac{d Q}{d \tau}+\frac{1}{\tau} \cdot \mathcal{E}_{\lambda}\right)-\frac{R(z)}{\Lambda \cdot C(q)} \cdot\left(\frac{C^{\prime}}{C(q)} \cdot \frac{d q}{d \tau}+\frac{1}{\tau} \cdot \mathcal{E}_{\Lambda}\right)=\frac{R^{\prime}(Z)}{\lambda \cdot C(Q)} \cdot \frac{d Z}{d \tau}+\frac{R^{\prime}(z)}{\Lambda \cdot C(q)} \cdot \frac{d z}{d \tau}$.

So we get the expressions via $\mathcal{E}_{\Lambda}, \mathcal{E}_{\lambda}, \mathcal{E}_{w}$, for the derivatives w.r.t. $\tau$ of $X, Y, Z, x, y, z$ :

$$
\begin{gathered}
\frac{d X}{d \tau}=\frac{1}{\tau} \cdot \frac{\mathcal{E}_{\Lambda}+\mathcal{E}_{w}}{\frac{R^{\prime \prime}(X)}{R^{\prime}(X)}}, \quad \frac{d Y}{d \tau}=\frac{1}{\tau} \cdot \frac{\mathcal{E}_{\Lambda}+\mathcal{E}_{w}+1}{\frac{R^{\prime \prime}(Y)}{R^{\prime}(Y)},} \quad \frac{d Z}{d \tau}=\frac{1}{\tau} \cdot \frac{\mathcal{E}_{\lambda}+\mathcal{E}_{w}+1}{\frac{R^{\prime \prime}(Z)}{R^{\prime}(Z)}}, \\
\frac{d x}{d \tau}=\frac{1}{\tau} \cdot \frac{\mathcal{E}_{\lambda}}{\frac{R^{\prime \prime}(x)}{R^{\prime}(x)}}, \quad \frac{d y}{d \tau}=\frac{1}{\tau} \cdot \frac{\mathcal{E}_{\lambda}+1}{\frac{R^{\prime \prime}(y)}{R^{\prime}(y)}}, \quad \frac{d z}{d \tau}=\frac{1}{\tau} \cdot \frac{\mathcal{E}_{\Lambda}+1}{\frac{R^{\prime \prime}(z)}{R^{\prime}(z)}}
\end{gathered}
$$

and three linear equations in $\mathcal{E}_{\Lambda}, \mathcal{E}_{\lambda}, \mathcal{E}_{w}$ :

$$
\begin{gathered}
L \cdot\left(\frac{R(X)}{\Lambda}+K \cdot \frac{R(Y)}{\Lambda}\right) \cdot\left(\mathcal{E}_{\Lambda}+\mathcal{E}_{w}\right)+(k+1) \cdot l \cdot \frac{R(Z)}{\lambda} \cdot\left(\mathcal{E}_{\lambda}+\mathcal{E}_{w}\right)+\frac{R^{\prime}(Z)}{\lambda} \cdot(K \cdot L \cdot Y+(k+1) \cdot l \cdot Z)=0 \\
(K+1) \cdot L \cdot \frac{R(z)}{\Lambda} \cdot \mathcal{E}_{\Lambda}+l \cdot\left(\frac{R(x)}{\lambda}+k \cdot \frac{R(y)}{\lambda}\right) \cdot \mathcal{E}_{\lambda}+\frac{R^{\prime}(z)}{\Lambda} \cdot(k \cdot l \cdot y+(K+1) \cdot L \cdot z)=0 \\
\frac{R(Z)}{\lambda \cdot C(Q)} \cdot\left(\frac{C^{\prime}}{C(Q)} \cdot \frac{d Q}{d \tau}+\frac{1}{\tau} \cdot \mathcal{E}_{\lambda}\right)-\frac{R(z)}{\Lambda \cdot C(q)} \cdot\left(\frac{C^{\prime}}{C(q)} \cdot \frac{d q}{d \tau}+\frac{1}{\tau} \cdot \mathcal{E}_{\Lambda}\right)=\frac{R^{\prime}(Z)}{\lambda \cdot C(Q)} \cdot \frac{d Z}{d \tau}+\frac{R^{\prime}(z)}{\Lambda \cdot C(q)} \cdot \frac{d z}{d \tau}
\end{gathered}
$$

Further,

$$
\mathcal{E}_{P^{X}}=\mathcal{E}_{\left(\frac{u^{\prime}(X)}{\Lambda}\right)}=\mathcal{E}_{u^{\prime}(X)}-\mathcal{E}_{\Lambda}=E_{u^{\prime}}(X) \cdot \mathcal{E}_{X}-\mathcal{E}_{\Lambda}=-r_{u}(X) \cdot \mathcal{E}_{X}-\mathcal{E}_{\Lambda}
$$

Considering the case $X \neq 0$ it is possible to write

$$
\mathcal{E}_{X}=\frac{\tau}{X} \cdot \frac{d X}{d \tau}=\frac{\tau}{X} \cdot \frac{1}{\tau} \cdot \frac{\mathcal{E}_{\Lambda}+\mathcal{E}_{w}}{\frac{R^{\prime \prime}(X)}{R^{\prime}(X)}}=-\frac{\mathcal{E}_{\Lambda}+\mathcal{E}_{w}}{r_{R}(X)}
$$

so that

$$
\mathcal{E}_{P^{X}}=\frac{r_{u}(X)}{r_{R}(X)} \cdot\left(\mathcal{E}_{\Lambda}+\mathcal{E}_{w}\right)-\mathcal{E}_{\Lambda}=\frac{r_{u}^{\prime}(X) \cdot X}{r_{R}(X) \cdot E_{R}(X)} \cdot \mathcal{E}_{\Lambda}+\frac{r_{u}(X)}{r_{R}(X)} \cdot \mathcal{E}_{w} .
$$

We have also

$$
\begin{aligned}
& \mathcal{E}_{P^{Y}}=\mathcal{E}_{\left(\frac{u^{\prime}(Y)}{\Lambda}\right)}=\mathcal{E}_{u^{\prime}(Y)}-\mathcal{E}_{\Lambda}=E_{u^{\prime}}(Y) \cdot \mathcal{E}_{Y}-\mathcal{E}_{\Lambda}=-r_{u}(Y) \cdot \mathcal{E}_{Y}-\mathcal{E}_{\Lambda}=\frac{r_{u}(Y)}{r_{R}(Y)} \cdot\left(\mathcal{E}_{\Lambda}+\mathcal{E}_{w}\right)+1-\mathcal{E}_{\Lambda}, \\
& \mathcal{E}_{P^{Z}}=\mathcal{E}_{\left(\frac{u^{\prime}(Z)}{\lambda}\right)}=\mathcal{E}_{u^{\prime}(Z)}-\mathcal{E}_{\lambda}=E_{u^{\prime}}(Z) \cdot \mathcal{E}_{Z}-\mathcal{E}_{\lambda}=-r_{u}(Z) \cdot \mathcal{E}_{Z}-\mathcal{E}_{\lambda}=\frac{r_{u}(Z)}{r_{R}(Z)} \cdot\left(\mathcal{E}_{\lambda}+\mathcal{E}_{w}+1\right)-\mathcal{E}_{\lambda}, \\
& \mathcal{E}_{p^{x}}=\mathcal{E}_{\left(\frac{u^{\prime}(x)}{\lambda}\right)}=\mathcal{E}_{u^{\prime}(x)}-\mathcal{E}_{\lambda}=E_{u^{\prime}}(x) \cdot \mathcal{E}_{x}-\mathcal{E}_{\lambda}=-r_{u}(x) \cdot \mathcal{E}_{x}-\mathcal{E}_{\lambda}=\frac{r_{u}(x)}{r_{R}(x)} \cdot \mathcal{E}_{\lambda}-\mathcal{E}_{\lambda}=-\frac{r_{u}^{\prime}(x) \cdot x}{r_{R}(x) \cdot E_{R}(x)} \cdot \mathcal{E}_{\lambda},
\end{aligned}
$$




$$
\begin{aligned}
& \mathcal{E}_{p^{y}}=\mathcal{E}_{\left(\frac{u^{\prime}(y)}{\lambda}\right)}=\mathcal{E}_{u^{\prime}(y)}-\mathcal{E}_{\lambda}=E_{u^{\prime}}(y) \cdot \mathcal{E}_{y}-\mathcal{E}_{\lambda}=-r_{u}(y) \cdot \mathcal{E}_{y}-\mathcal{E}_{\lambda}=\frac{r_{u}(y)}{r_{R}(y)} \cdot\left(\mathcal{E}_{\lambda}+1\right)-\mathcal{E}_{\lambda}, \\
& \mathcal{E}_{p^{z}}=\mathcal{E}_{\left(\frac{u^{\prime}(z)}{\Lambda}\right)}=\mathcal{E}_{u^{\prime}(z)}-\mathcal{E}_{\Lambda}=E_{u^{\prime}}(z) \cdot \mathcal{E}_{z}-\mathcal{E}_{\Lambda}=-r_{u}(z) \cdot \mathcal{E}_{z}-\mathcal{E}_{\Lambda}=\frac{r_{u}(z)}{r_{R}(z)} \cdot\left(\mathcal{E}_{\Lambda}+1\right)-\mathcal{E}_{\Lambda} .
\end{aligned}
$$

\subsection{Proof of Lemma 2}

From Lemma 1 we have

$$
\begin{gathered}
\frac{d X}{d \tau}=-\frac{X}{r_{R}} \cdot\left(\mathcal{E}_{\Lambda}+\mathcal{E}_{w}\right), \quad \frac{d Y}{d \tau}=-\frac{X}{r_{R}} \cdot\left(\mathcal{E}_{\Lambda}+\mathcal{E}_{w}+1\right), \quad \frac{d Z}{d \tau}=-\frac{X}{r_{R}} \cdot\left(\mathcal{E}_{\lambda}+\mathcal{E}_{w}+1\right), \\
\frac{d x}{d \tau}=-\frac{X}{r_{R}} \cdot \mathcal{E}_{\lambda}, \quad \frac{d y}{d \tau}=-\frac{X}{r_{R}} \cdot\left(\mathcal{E}_{\lambda}+1\right), \quad \frac{d z}{d \tau}=-\frac{X}{r_{R}} \cdot\left(\mathcal{E}_{\Lambda}+1\right) .
\end{gathered}
$$

Moreover,

$$
\begin{gathered}
\frac{d Q}{d \tau}=L \cdot \frac{d X}{d \tau}+K \cdot L \cdot \frac{d Y}{d \tau}+(k+1) \cdot l \cdot \frac{d Z}{d \tau}+Q-L \cdot X= \\
=-\frac{X}{r_{R}} \cdot\left(L \cdot\left(\mathcal{E}_{\Lambda}+\mathcal{E}_{w}\right)+K \cdot L \cdot\left(\mathcal{E}_{\Lambda}+\mathcal{E}_{w}+1\right)+(k+1) \cdot l \cdot\left(\mathcal{E}_{\lambda}+\mathcal{E}_{w}+1\right)\right)+Q-L \cdot X= \\
=-\frac{X}{r_{R}} \cdot\left(L \cdot\left(\mathcal{E}_{\Lambda}+\mathcal{E}_{w}\right)+K \cdot L \cdot\left(\mathcal{E}_{\Lambda}+\mathcal{E}_{w}\right)+K \cdot L+(k+1) \cdot l \cdot\left(\mathcal{E}_{\lambda}+\mathcal{E}_{w}\right)+(k+1) \cdot l\right)+Q-L \cdot X= \\
=-\frac{X}{r_{R}} \cdot\left((K+1) \cdot L \cdot\left(\mathcal{E}_{\Lambda}+\mathcal{E}_{w}\right)+(k+1) \cdot l \cdot\left(\mathcal{E}_{\lambda}+\mathcal{E}_{w}\right)+K \cdot L+(k+1) \cdot l\right)+Q-L \cdot X= \\
=-\frac{X}{r_{R}} \cdot\left(-(K \cdot L+(k+1) \cdot l) \cdot E_{R}+K \cdot L+(k+1) \cdot l\right)+Q-L \cdot X= \\
=-\frac{X}{r_{R}} \cdot(K \cdot L+(k+1) \cdot l) \cdot\left(1-E_{R}\right)+Q-L \cdot X= \\
=(\Gamma-L) \cdot \frac{r_{R}+E_{R}-1}{r_{R}} \cdot X=(\Gamma-L) \cdot \frac{r_{u}^{\prime} \cdot X^{2}}{r_{R} \cdot E_{R}} \\
\frac{d q}{d \tau}=l \cdot \frac{d x}{d \tau}+k \cdot l \cdot \frac{d y}{d \tau}+(K+1) \cdot L \cdot \frac{d z}{d \tau}+Q-l \cdot X= \\
=-\frac{X}{r_{R}} \cdot\left(l \cdot \mathcal{E}_{\lambda}+k \cdot l \cdot\left(\mathcal{E}_{\lambda}+1\right)+(K+1) \cdot L \cdot\left(\mathcal{E}_{\Lambda}+1\right)\right)+Q-l \cdot X= \\
=-\frac{X}{r_{R}} \cdot\left((k+1) \cdot l \cdot \mathcal{E}_{\lambda}+(K+1) \cdot L \cdot \mathcal{E}_{\Lambda}+(K+1) \cdot L+k \cdot l\right)+Q-l \cdot X= \\
=-\frac{X}{r_{R}} \cdot\left(-(k \cdot l+(K+1) \cdot L) \cdot E_{R}+(K+1) \cdot L+k \cdot l\right)+Q-l \cdot X= \\
=-\frac{X}{r_{R}} \cdot\left((k \cdot l+(K+1) \cdot L) \cdot\left(1-\mathcal{E}_{R}\right)\right)+Q-l \cdot X= \\
=-\frac{X}{r_{R}} \cdot(\Gamma-l) \cdot\left(1-E_{R}\right)+(\Gamma-l) \cdot X=(\Gamma-l) \cdot \frac{E_{R}+r_{R}-1}{r_{R}} \cdot X=(\Gamma-l) \cdot \frac{r_{u}^{\prime} \cdot X^{2}}{r_{R} \cdot \mathcal{E}_{R}} \\
\end{gathered}
$$


For what concerns the last three equations for Comparative Statics, they can be written as

$$
\begin{gathered}
L \cdot(1+K) \cdot\left(\mathcal{E}_{\Lambda}+\mathcal{E}_{w}\right)+(k+1) \cdot l \cdot\left(\mathcal{E}_{\lambda}+\mathcal{E}_{w}\right)+(K \cdot L+(k+1) \cdot l) \cdot \mathcal{E}_{R}=0, \\
L \cdot(1+K) \cdot \mathcal{E}_{\Lambda}+(k+1) \cdot l \cdot \mathcal{E}_{\lambda}+(k \cdot l+(K+1) \cdot L) \cdot \mathcal{E}_{R}=0 \\
\left(\frac{C^{\prime}}{C} \cdot \frac{d Q}{d \tau}+\mathcal{E}_{\lambda}\right)-\left(\frac{C^{\prime}}{C} \cdot \frac{d q}{d \tau}+\mathcal{E}_{\Lambda}\right)-\frac{R^{\prime}}{R} \cdot \frac{d Z}{d \tau}+\frac{R^{\prime}}{R} \cdot \frac{d z}{d \tau}=0
\end{gathered}
$$

i.e.,

$$
\begin{gathered}
\mathcal{E}_{w}=\frac{L-l}{\Gamma} \cdot E_{R}(>0), \\
(K+1) \cdot L \cdot \mathcal{E}_{\Lambda}+l \cdot(1+k) \cdot \mathcal{E}_{\lambda}=. \\
-(\Gamma-l) \cdot E_{R}\left(\frac{C^{\prime}}{C} \cdot(\Gamma-L) \cdot \frac{r_{u}^{\prime} \cdot X^{2}}{r_{R} \cdot E_{R}}+\mathcal{E}_{\lambda}\right)-\left(\frac{C^{\prime}}{C} \cdot(\Gamma-l) \cdot \frac{r_{u}^{\prime} \cdot X^{2}}{r_{R} \cdot E_{R}}+\mathcal{E}_{\Lambda}\right)+\frac{E_{R}}{r_{R}} \cdot\left(\mathcal{E}_{\lambda}+\mathcal{E}_{w}+1\right)= \\
=\frac{E_{R}}{r_{R}} \cdot\left(\mathcal{E}_{\Lambda}+1\right) .
\end{gathered}
$$

i.e.,

$$
\begin{gathered}
\mathcal{E}_{w}=\frac{L-l}{\Gamma} \cdot E_{R}(>0), \\
\mathcal{E}_{\Lambda}=-\left(l \cdot(k+1) \cdot \frac{L-l}{\Gamma} \cdot \frac{r_{R}-1}{E_{R}+r_{R}}+\Gamma-l\right) \cdot \frac{E_{R}}{\Gamma}, \\
\mathcal{E}_{\lambda}=-\left(-L \cdot(K+1) \cdot \frac{L-l}{\Gamma} \cdot \frac{r_{R}-1}{E_{R}+r_{R}}+\Gamma-l\right) \cdot \frac{E_{R}}{\Gamma} .
\end{gathered}
$$

Further,

$$
\begin{gathered}
\mathcal{E}_{\Lambda}=-\left(l \cdot(k+1) \cdot \frac{L-l}{\Gamma} \cdot \frac{r_{R}+E_{R}-1-E_{R}}{E_{R}+r_{R}}+\Gamma-l\right) \cdot \frac{E_{R}}{\Gamma}= \\
=-\left(l \cdot(k+1) \cdot \frac{L-l}{\Gamma} \cdot \frac{r_{R}+E_{R}-1}{E_{R}+r_{R}}-l \cdot(k+1) \cdot \frac{L-l}{\Gamma} \cdot \frac{E_{R}}{E_{R}+r_{R}}+\Gamma-l\right) \cdot \frac{E_{R}}{\Gamma}= \\
=-l \cdot(k+1) \cdot \frac{L-l}{\Gamma} \cdot \frac{r_{u}^{\prime} \cdot X}{\left(E_{R}+r_{R}\right) \cdot \mathcal{E}_{R}} \cdot \frac{E_{R}}{\Gamma}-\frac{-l \cdot(k+1) \cdot(L-l) \cdot E_{R}+\Gamma \cdot(\Gamma-l) \cdot\left(E_{R}+r_{R}\right)}{\Gamma \cdot\left(E_{R}+r_{R}\right)} \cdot \frac{E_{R}}{\Gamma}= \\
=-l \cdot(k+1) \cdot \frac{L-l}{\Gamma} \cdot \frac{r_{u}^{\prime} \cdot X}{\Gamma \cdot\left(\mathcal{E}_{R}+r_{R}\right)}-\frac{(L \cdot(K+1) \cdot(\Gamma-l)+l \cdot(k+1) \cdot(\Gamma-L)) \cdot E_{R}+\Gamma \cdot(\Gamma-l) \cdot r_{R}}{\Gamma \cdot\left(E_{R}+r_{R}\right)} \cdot \frac{E_{R}}{\Gamma}, \\
\mathcal{E}_{\Lambda}+\mathcal{E}_{w}=-\left(l \cdot(k+1) \cdot \frac{L-l}{\Gamma} \cdot \frac{r_{R}-1}{E_{R}+r_{R}}+\Gamma-L\right) \cdot \frac{E_{R}}{\Gamma}= \\
=-\left(l \cdot(k+1) \cdot \frac{L-l}{\Gamma} \cdot \frac{r_{R}+E_{R}-1-E_{R}}{E_{R}+r_{R}}+\Gamma-L\right) \cdot \frac{E_{R}}{\Gamma}= \\
=-\left(l \cdot(k+1) \cdot \frac{L-l}{\Gamma} \cdot \frac{r_{R}+E_{R}-1}{E_{R}+r_{R}}-l \cdot(k+1) \cdot \frac{L-l}{\Gamma} \cdot \frac{E_{R}}{E_{R}+r_{R}}+\Gamma-L\right) \cdot \frac{E_{R}}{\Gamma}= \\
=-\left(l \cdot(k+1) \cdot \frac{L-l}{\Gamma} \cdot \frac{r_{u}^{\prime} \cdot X}{\left(E_{R}+r_{R}\right) \cdot E_{R}}+\frac{-l \cdot(k+1) \cdot(L-l) \cdot E_{R}+\Gamma \cdot(\Gamma-L) \cdot\left(E_{R}+r_{R}\right)}{\Gamma \cdot\left(E_{R}+r_{R}\right)}\right) \cdot \frac{E_{R}}{\Gamma}=
\end{gathered}
$$




$$
\begin{aligned}
& =-l \cdot(k+1) \cdot \frac{L-l}{\Gamma} \cdot \frac{r_{u}^{\prime} \cdot X}{\left(E_{R}+r_{R}\right) \cdot E_{R}} \cdot \frac{E_{R}}{\Gamma}-\frac{(-l \cdot(k+1) \cdot(L-l)+\Gamma \cdot(\Gamma-L)) \cdot E_{R}+\Gamma \cdot(\Gamma-L) \cdot r_{R}}{\Gamma \cdot\left(E_{R}+r_{R}\right)} \cdot \frac{E_{R}}{\Gamma}= \\
& =-l \cdot(k+1) \cdot \frac{L-l}{\Gamma} \cdot \frac{r_{u}^{\prime} \cdot X}{\left(E_{R}+r_{R}\right) \cdot E_{R}} \cdot \frac{E_{R}}{\Gamma}-\frac{(l \cdot(k+1) \cdot(-L+l+\Gamma)+\Gamma \cdot L \cdot K) \cdot E_{R}+\Gamma \cdot(\Gamma-L) \cdot r_{R}}{\Gamma \cdot\left(E_{R}+r_{R}\right)} \cdot \frac{E_{R}}{\Gamma}= \\
& =-l \cdot(k+1) \cdot \frac{L-l}{\Gamma} \cdot \frac{r_{u}^{\prime} \cdot X}{\left(E_{R}+r_{R}\right) \cdot E_{R}} \cdot \frac{E_{R}}{\Gamma}-\frac{(l \cdot(k+1) \cdot(L \cdot K+l \cdot(k+2))+\Gamma \cdot L \cdot K) \cdot E_{R}+\Gamma \cdot(\Gamma-L) \cdot r_{R}}{\Gamma \cdot\left(E_{R}+r_{R}\right)} \cdot \frac{E_{R}}{\Gamma} .
\end{aligned}
$$

Hence if $r_{u}^{\prime} \geq 0$ then $\mathcal{E}_{\Lambda}<\mathcal{E}_{\Lambda}+\mathcal{E}_{w}<0$.

Moreover:

$$
\begin{gathered}
\mathcal{E}_{\lambda}=-\left(-L \cdot(K+1) \cdot \frac{L-l}{\Gamma} \cdot \frac{r_{R}+E_{R}-E_{R}-1}{E_{R}+r_{R}}+\Gamma-l\right) \cdot \frac{E_{R}}{\Gamma}= \\
=-\left(-L \cdot(K+1) \cdot \frac{L-l}{\Gamma}+L \cdot(K+1) \cdot \frac{L-l}{\Gamma} \cdot \frac{E_{R}+1}{E_{R}+r_{R}}+\Gamma-l\right) \cdot \frac{E_{R}}{\Gamma}= \\
=-\left(\frac{\Gamma \cdot(\Gamma-l)-L \cdot(K+1) \cdot(L-l)}{\Gamma}+L \cdot(K+1) \cdot \frac{L-l}{\Gamma} \cdot \frac{E_{R}+1}{E_{R}+r_{R}}\right) \cdot \frac{E_{R}}{\Gamma}= \\
=-\left(\frac{L \cdot(K+1) \cdot(\Gamma-L)+l \cdot(k+1) \cdot(\Gamma-l)}{\Gamma}+L \cdot(K+1) \cdot \frac{L-l}{\Gamma} \cdot \frac{E_{R}+1}{E_{R}+r_{R}}\right) \cdot \frac{E_{R}}{\Gamma}
\end{gathered}
$$

so that $\mathcal{E}_{\lambda}<0$. Further,

$$
\begin{gathered}
\mathcal{E}_{N}=-\mathcal{E}_{C(Q)}=-E_{C} \cdot \mathcal{E}_{Q}=-E_{C} \cdot Q \cdot(\Gamma-L) \cdot \frac{r_{u}^{\prime} \cdot X^{2}}{r_{R} \cdot E_{R}}=-Q \cdot(\Gamma-L) \cdot \frac{r_{u}^{\prime} \cdot X^{2}}{r_{R}}=-\Gamma \cdot(\Gamma-L) \cdot \frac{r_{u}^{\prime} \cdot X^{3}}{r_{R}}, \\
\frac{d N}{d \tau}=-N \cdot \Gamma \cdot(\Gamma-L) \cdot \frac{r_{u}^{\prime} \cdot X^{3}}{r_{R}}=-L \cdot \Gamma \cdot(\Gamma-L) \cdot \frac{r_{u}^{\prime} \cdot X^{3}}{C \cdot r_{R}}, \\
\mathcal{E}_{n}=-\mathcal{E}_{C(q)}=-E_{C} \cdot \mathcal{E}_{q}=-E_{C} \cdot Q \cdot(\Gamma-l) \cdot \frac{r_{u}^{\prime} \cdot X^{2}}{r_{R} \cdot E_{R}}=-Q \cdot(\Gamma-l) \cdot \frac{r_{u}^{\prime} \cdot X^{2}}{r_{R}}=-\Gamma \cdot(\Gamma-l) \cdot \frac{r_{u}^{\prime} \cdot X^{3}}{r_{R}}, \\
\frac{d n}{d \tau}=-n \cdot \Gamma \cdot(\Gamma-l) \cdot \frac{r_{u}^{\prime} \cdot X^{3}}{r_{R}}=-l \cdot \Gamma \cdot(\Gamma-l) \cdot \frac{r_{u}^{\prime} \cdot X^{3}}{C \cdot r_{R}} .
\end{gathered}
$$

\subsection{Proof of Lemma 3}

We have

$$
\frac{W^{B}}{L}=L \cdot \frac{u(X)+K \cdot u(Y)}{C(Q)}+l \cdot(k+1) \cdot \frac{u(z)}{C(q)}
$$

Hence

$$
\frac{1}{L} \cdot \frac{\partial W^{B}}{\partial \tau}=L \cdot\left(\frac{u^{\prime}(X) \cdot \frac{d X}{d \tau}+K \cdot u^{\prime}(Y) \cdot \frac{d Y}{d \tau}}{C(Q)}-\frac{u(X)+K \cdot u(Y)}{(C(Q))^{2}} \cdot C^{\prime}(Q) \cdot \frac{d Q}{d \tau}\right)+
$$




$$
\begin{gathered}
+l \cdot(k+1) \cdot\left(\frac{u^{\prime}(z) \cdot \frac{d z}{d \tau}}{C(q)}-\frac{u(z)}{(C(q))^{2}} \cdot C^{\prime}(q) \cdot \frac{d q}{d \tau}\right)= \\
=L \cdot\left(\frac{u^{\prime}}{C} \cdot\left(\frac{d X}{d \tau}+K \cdot \frac{d Y}{d \tau}\right)-(1+K) \cdot \frac{u \cdot C^{\prime}}{C^{2}} \cdot \frac{d Q}{d \tau}\right)+l \cdot(k+1) \cdot\left(\frac{u^{\prime}}{C} \cdot \frac{d z}{d \tau}-\frac{u \cdot C^{\prime}}{C^{2}} \cdot \frac{d q}{d \tau}\right)= \\
=\frac{u^{\prime}}{C} \cdot\left(L \cdot \frac{d X}{d \tau}+K \cdot L \cdot \frac{d Y}{d \tau}+l \cdot(k+1) \cdot \frac{d z}{d \tau}\right)-\frac{u \cdot C^{\prime}}{C^{2}} \cdot\left(L \cdot(K+1) \cdot \frac{d Q}{d \tau}+l \cdot(k+1) \cdot \frac{d q}{d \tau}\right)
\end{gathered}
$$

Since

$$
\begin{gathered}
\frac{d X}{d \tau}=-\frac{X}{r_{R}} \cdot\left(\mathcal{E}_{\Lambda}+\mathcal{E}_{w}\right), \quad \frac{d Y}{d \tau}=-\frac{X}{r_{R}} \cdot\left(\mathcal{E}_{\Lambda}+\mathcal{E}_{w}+1\right), \quad \frac{d z}{d \tau}=-\frac{X}{r_{R}} \cdot\left(\mathcal{E}_{\Lambda}+1\right), \\
\frac{d Q}{d \tau}=(\Gamma-L) \cdot \frac{r_{u}^{\prime} \cdot X^{2}}{r_{R} \cdot E_{R}}, \quad \frac{d q}{d \tau}=(\Gamma-l) \cdot \frac{r_{u}^{\prime} \cdot X^{2}}{r_{R} \cdot E_{R}}
\end{gathered}
$$

from the last expression we can write

$$
\begin{gathered}
\frac{1}{L} \cdot \frac{\partial W^{B}}{\partial \tau}=-\frac{X}{r_{R}} \cdot \frac{u^{\prime}}{C} \cdot\left(L \cdot\left(\mathcal{E}_{\Lambda}+\mathcal{E}_{w}\right)+K \cdot L \cdot\left(\mathcal{E}_{\Lambda}+\mathcal{E}_{w}+1\right)+l \cdot(k+1) \cdot\left(\mathcal{E}_{\Lambda}+1\right)\right)- \\
-\frac{u \cdot C^{\prime}}{C^{2}} \cdot \frac{r_{u}^{\prime} \cdot X^{2}}{r_{R} \cdot E_{R}} \cdot(L \cdot(K+1) \cdot(\Gamma-L)+l \cdot(k+1) \cdot(\Gamma-l))= \\
=-\frac{X}{r_{R}} \cdot \frac{u^{\prime}}{C} \cdot\left(\Gamma \cdot \mathcal{E}_{\Lambda}+L \cdot(K+1) \cdot \mathcal{E}_{w}+\Gamma-L\right)- \\
-\frac{u \cdot C^{\prime} \cdot Q}{\Gamma \cdot C^{2}} \cdot \frac{r_{u}^{\prime} \cdot X}{r_{R} \cdot E_{R}} \cdot(L \cdot(K+1) \cdot(\Gamma-L)+l \cdot(k+1) \cdot(\Gamma-l)) .
\end{gathered}
$$

Moreover, since

$$
\mathcal{E}_{\Lambda}=-\left(l \cdot(k+1) \cdot \frac{L-l}{\Gamma} \cdot \frac{r_{R}-1}{E_{R}+r_{R}}+\Gamma-l\right) \cdot \frac{E_{R}}{\Gamma}, \quad \mathcal{E}_{w}=(L-l) \cdot \frac{E_{R}}{\Gamma}
$$

we can also write

$$
\begin{gathered}
\frac{1}{L} \cdot \frac{\partial W^{B}}{\partial \tau}=-\frac{X}{r_{R}} \cdot \frac{u^{\prime}}{C} \cdot\left(\Gamma \cdot\left(-\frac{l \cdot(k+1)}{\Gamma} \cdot \frac{L-l}{\Gamma} \cdot \frac{r_{R}-1}{E_{R}+r_{R}} \cdot E_{R}-\frac{\Gamma-l}{\Gamma} \cdot E_{R}\right)+\right. \\
\left.+L \cdot(K+1) \cdot \frac{L-l}{\Gamma} \cdot E_{R}+\Gamma-L\right)-\frac{u \cdot E_{C}}{\Gamma \cdot C} \cdot \frac{r_{u}^{\prime} \cdot X}{r_{R} \cdot E_{R}} \cdot(L \cdot(K+1) \cdot(\Gamma-L)+l \cdot(k+1) \cdot(\Gamma-l))= \\
=-\frac{X}{r_{R}} \cdot \frac{u^{\prime}}{C} \cdot\left(-l \cdot(k+1) \cdot \frac{L-l}{\Gamma} \cdot \frac{r_{R}-1}{E_{R}+r_{R}} \cdot E_{R}+\left(L \cdot(K+1) \cdot \frac{L-l}{\Gamma}-(\Gamma-l)\right) \cdot E_{R}+\Gamma-L\right)- \\
-\frac{u}{\Gamma \cdot C} \cdot \frac{r_{u}^{\prime} \cdot X}{r_{R}} \cdot(L \cdot(K+1) \cdot(\Gamma-L)+l \cdot(k+1) \cdot(\Gamma-l))= \\
=-\frac{X}{r_{R}} \cdot \frac{u^{\prime}}{C} \cdot\left(L \cdot(K+1) \cdot \frac{L-l}{\Gamma}-(\Gamma-l)\right) \cdot E_{R}-\frac{X}{r_{R}} \cdot \frac{u^{\prime}}{C} \cdot\left(-l \cdot(k+1) \cdot \frac{L-l}{\Gamma} \cdot \frac{r_{R}-1}{E_{R}+r_{R}} \cdot E_{R}+\Gamma-L\right)- \\
-\frac{u}{\Gamma \cdot C} \cdot \frac{r_{u}^{\prime} \cdot X}{r_{R}} \cdot(L \cdot(K+1) \cdot(\Gamma-L)+l \cdot(k+1) \cdot(\Gamma-l))=
\end{gathered}
$$




$$
\begin{aligned}
& =-\frac{X}{r_{R}} \cdot \frac{u^{\prime}}{C} \cdot \frac{\left(L \cdot(K+1) \cdot \frac{L-l}{\Gamma}-(\Gamma-l)\right) \cdot E_{R} \cdot\left(E_{R}+r_{R}-1+1\right)}{E_{R}+r_{R}}- \\
& -\frac{X}{r_{R}} \cdot \frac{u^{\prime}}{C} \cdot \frac{-l \cdot(k+1) \cdot \frac{L-l}{\Gamma} \cdot\left(r_{R}-1\right) \cdot E_{R}+(\Gamma-L) \cdot\left(E_{R}+r_{R}\right)}{E_{R}+r_{R}}- \\
& -\frac{u}{\Gamma \cdot C} \cdot \frac{r_{u}^{\prime} \cdot X}{r_{R}} \cdot(L \cdot(K+1) \cdot(\Gamma-L)+l \cdot(k+1) \cdot(\Gamma-l))= \\
& =-\frac{X}{r_{R}} \cdot \frac{u^{\prime}}{C} \cdot \frac{\left(L \cdot(K+1) \cdot \frac{L-l}{\Gamma}-(\Gamma-l)\right) \cdot E_{R} \cdot\left(E_{R}+r_{R}-1\right)}{E_{R}+r_{R}}- \\
& -\frac{X}{r_{R}} \cdot \frac{u^{\prime}}{C} \cdot \frac{\left(L \cdot(K+1) \cdot \frac{L-l}{\Gamma}-(\Gamma-l)\right) \cdot E_{R}}{E_{R}+r_{R}}- \\
& -\frac{X}{r_{R}} \cdot \frac{u^{\prime}}{C} \cdot \frac{l \cdot(k+1) \cdot \frac{L-l}{\Gamma} \cdot E_{R}-l \cdot(k+1) \cdot \frac{L-l}{\Gamma} \cdot E_{R} \cdot r_{R}+(\Gamma-L) \cdot\left(E_{R}+r_{R}\right)}{E_{R}+r_{R}}- \\
& -\frac{u}{\Gamma \cdot C} \cdot \frac{r_{u}^{\prime} \cdot X}{r_{R}} \cdot(L \cdot(K+1) \cdot(\Gamma-L)+l \cdot(k+1) \cdot(\Gamma-l))= \\
& =-\frac{X}{r_{R}} \cdot \frac{u^{\prime}}{C} \cdot \frac{\left(L \cdot(K+1) \cdot \frac{L-l}{\Gamma}-(\Gamma-l)\right) \cdot r_{u}^{\prime} \cdot X}{E_{R}+r_{R}}- \\
& -\frac{X}{r_{R}} \cdot \frac{u^{\prime}}{C} \cdot \frac{\left(L \cdot(K+1) \cdot \frac{L-l}{\Gamma}-(\Gamma-l)\right) \cdot E_{R}}{E_{R}+r_{R}}-\frac{X}{r_{R}} \cdot \frac{u^{\prime}}{C} \cdot \frac{\left(l \cdot(k+1) \cdot \frac{L-l}{\Gamma}+\Gamma-L\right) \cdot E_{R}}{E_{R}+r_{R}}- \\
& -\frac{X}{r_{R}} \cdot \frac{u^{\prime}}{C} \cdot \frac{\left(\Gamma-L-l \cdot(k+1) \cdot \frac{L-l}{\Gamma} \cdot E_{R}\right) \cdot r_{R}}{E_{R}+r_{R}}- \\
& -\frac{u}{\Gamma \cdot C} \cdot \frac{r_{u}^{\prime} \cdot X}{r_{R}} \cdot(L \cdot(K+1) \cdot(\Gamma-L)+l \cdot(k+1) \cdot(\Gamma-l))= \\
& \text { (since } \left.\left(L \cdot(K+1) \cdot \frac{L-l}{\Gamma}-(\Gamma-l)\right) \cdot E_{R}+\left(l \cdot(k+1) \cdot \frac{L-l}{\Gamma}+\Gamma-L\right) \cdot E_{R}=0 \text { and } E_{R}=1-r_{u}\right) \\
& =-\frac{X}{r_{R}} \cdot \frac{u^{\prime}}{\Gamma \cdot C} \cdot \frac{(L \cdot(K+1) \cdot(L-l)-\Gamma \cdot(\Gamma-l)) \cdot r_{u}^{\prime} \cdot X}{E_{R}+r_{R}}- \\
& -\frac{X}{r_{R}} \cdot \frac{u^{\prime}}{C} \cdot \frac{\left(\Gamma-L-l \cdot(k+1) \cdot \frac{L-l}{\Gamma}+l \cdot(k+1) \cdot \frac{L-l}{\Gamma} \cdot r_{u}\right)}{E_{R}+r_{R}} \cdot r_{R^{-}} \\
& -\frac{u}{\Gamma \cdot C} \cdot \frac{r_{u}^{\prime} \cdot X}{r_{R}} \cdot(L \cdot(K+1) \cdot(\Gamma-L)+l \cdot(k+1) \cdot(\Gamma-l))= \\
& \text { (since } \Gamma \cdot(\Gamma-L)-l \cdot(k+1) \cdot(L-l)=\Gamma \cdot L \cdot K+l \cdot(k+1) \cdot(\Gamma-L+l) \text { ) } \\
& =\frac{X}{r_{R}} \cdot \frac{u^{\prime} \cdot r_{u}^{\prime} \cdot X}{\Gamma \cdot C} \cdot \frac{(L \cdot(K+1) \cdot(\Gamma-L)+l \cdot(k+1) \cdot(\Gamma-l))}{E_{R}+r_{R}}-
\end{aligned}
$$




$$
\begin{aligned}
&- \frac{X}{r_{R}} \cdot \frac{u^{\prime}}{\Gamma \cdot C} \cdot \frac{\left(\Gamma \cdot L \cdot K+l \cdot(k+1) \cdot(\Gamma-L+l)+l \cdot(k+1) \cdot(L-l) \cdot r_{u}\right)}{E_{R}+r_{R}} \cdot r_{R}- \\
&-\frac{u}{\Gamma \cdot C} \cdot \frac{r_{u}^{\prime} \cdot X}{r_{R}} \cdot(L \cdot(K+1) \cdot(\Gamma-L)+l \cdot(k+1) \cdot(\Gamma-l))= \\
&=-\frac{X}{r_{R}} \cdot \frac{u^{\prime}}{\Gamma \cdot C} \cdot \frac{\Gamma \cdot L \cdot K+l \cdot(k+1) \cdot(\Gamma-L+l)+l \cdot(k+1) \cdot(L-l) \cdot r_{u}}{E_{R}+r_{R}} \cdot r_{R}+ \\
&+\frac{(L \cdot(K+1) \cdot(\Gamma-L)+l \cdot(k+1) \cdot(\Gamma-l))}{\mathcal{E}_{R}+r_{R}} \cdot \frac{r_{u}^{\prime} \cdot X \cdot u}{r_{R} \cdot \Gamma \cdot C} \cdot\left(E_{u}-\left(E_{R}+r_{R}\right)\right)= \\
&=-\frac{X}{r_{R}} \cdot \frac{u^{\prime}}{\Gamma \cdot C} \cdot \frac{\Gamma \cdot L \cdot K+l \cdot(k+1) \cdot(\Gamma-L+l)+l \cdot(k+1) \cdot(L-l) \cdot r_{u}}{E_{R}+r_{R}} \cdot r_{R}+ \\
&+ \frac{(L \cdot(K+1) \cdot(\Gamma-L)+l \cdot(k+1) \cdot(\Gamma-l))}{\mathcal{E}_{R}+r_{R}} \cdot \frac{r_{u}^{\prime} \cdot X \cdot u}{r_{R} \cdot \Gamma \cdot C} \cdot\left(E_{u}-1-\left(E_{R}+r_{R}-1\right)\right)= \\
&=-\frac{u \cdot \mathcal{E}_{u}}{r_{R} \cdot \Gamma \cdot C} \cdot \frac{\Gamma \cdot L \cdot K+l \cdot(k+1) \cdot(\Gamma-L+l)+l \cdot(k+1) \cdot(L-l) \cdot r_{u}}{E_{R}+r_{R}} \cdot r_{R}- \\
&- \frac{(L \cdot(K+1) \cdot(\Gamma-L)+l \cdot(k+1) \cdot(\Gamma-l))}{\mathcal{E}_{R}+r_{R}} \cdot \frac{r_{u}^{\prime} \cdot X \cdot u}{r_{R} \cdot \Gamma \cdot C} \cdot\left(1-E_{u}+\frac{r_{u}^{\prime} \cdot X}{E_{R}}\right)= \\
&=-\frac{u}{\Gamma \cdot C \cdot r_{R}} \cdot\left(A^{B}+B^{B} \cdot r_{u}^{\prime} \cdot X\right),
\end{aligned}
$$

where

$$
\begin{gathered}
A^{B}=\left(\Gamma \cdot L \cdot K+l \cdot(k+1) \cdot\left(\Gamma-L+l+(L-l) \cdot r_{u}\right)\right) \cdot \frac{r_{R} \cdot E_{u}}{E_{R}+r_{R}}(>0), \\
B^{B}=(L \cdot(K+1) \cdot(\Gamma-L)+l \cdot(k+1) \cdot(\Gamma-l)) \cdot \frac{\left(\left(1-E_{u}\right) \cdot E_{R}+r_{u}^{\prime} \cdot X\right)}{\left(E_{R}+r_{R}\right) \cdot E_{R}} .
\end{gathered}
$$

Hence if $r_{u}^{\prime} \geq 0$ then $\frac{d W^{B}}{d \tau}>0$, since $E_{u}<1$.

Further,

$$
\frac{W^{s}}{l}=n \cdot(u(x)+k \cdot l \cdot u(y))+N \cdot(K+1) u(Z)=l \cdot \frac{u(x)+k \cdot u(y)}{C(q)}+L \cdot(K+1) \cdot \frac{u(Z)}{C(Q)} .
$$

Hence

$$
\begin{aligned}
& \frac{1}{l} \cdot \frac{d W^{s}}{d \tau}=l \cdot\left(\frac{u^{\prime}(x) \cdot \frac{d x}{d \tau}+k \cdot u^{\prime}(y) \cdot \frac{d y}{d \tau}}{C(q)}-\frac{u(x)+k \cdot u(y)}{(C(q))^{2}} \cdot C^{\prime}(q) \cdot \frac{d q}{d \tau}\right)+ \\
&+L \cdot(K+1) \cdot\left(\frac{u^{\prime}(Z) \cdot \frac{d Z}{d \tau}}{C(Q)}-\frac{u(Z)}{(C(Q))^{2}} \cdot C^{\prime}(Q) \cdot \frac{d Q}{d \tau}\right)= \\
&=l \cdot \frac{u^{\prime}(x) \cdot \frac{d x}{d \tau}+k \cdot u^{\prime}(y) \cdot \frac{d y}{d \tau}}{C(q)}+L \cdot(K+1) \cdot \frac{u^{\prime}(Z) \cdot \frac{d Z}{d \tau}}{C(Q)}- \\
&-\left(l \cdot \frac{u(x)+k \cdot u(y)}{(C(q))^{2}} \cdot C^{\prime}(q) \cdot \frac{d q}{d \tau}+L \cdot(K+1) \cdot \frac{u(Z)}{(C(Q))^{2}} \cdot C^{\prime}(Q) \cdot \frac{d Q}{d \tau}\right)=
\end{aligned}
$$




$$
=\frac{u^{\prime}}{C} \cdot\left(l \cdot\left(\frac{d x}{d \tau}+k \cdot \frac{d y}{d \tau}\right)+L \cdot(K+1) \cdot \frac{d Z}{d \tau}\right)-\frac{u \cdot C^{\prime}}{C^{2}} \cdot\left(l \cdot(k+1) \cdot \frac{d q}{d \tau}+L \cdot(K+1) \cdot \frac{d Q}{d \tau}\right) .
$$

The last expression, since

$$
\frac{d x}{d \tau}=-\frac{X}{r_{R}} \cdot \mathcal{E}_{\lambda}, \frac{d y}{d \tau}=-\frac{X}{r_{R}} \cdot\left(\mathcal{E}_{\lambda}+1\right), \frac{d Z}{d \tau}=-\frac{X}{r_{R}} \cdot\left(\mathcal{E}_{\lambda}+\mathcal{E}_{w}+1\right),
$$

and

can be rewritten as

$$
\frac{d Q}{d \tau}=(\Gamma-L) \cdot \frac{r_{u}^{\prime} \cdot X^{2}}{r_{R} \cdot E_{R}}, \frac{d q}{d \tau}=(\Gamma-l) \cdot \frac{r_{u}^{\prime} \cdot X^{2}}{r_{R} \cdot E_{R}}
$$

$$
\begin{aligned}
& -\frac{X}{r_{R}} \cdot \frac{u^{\prime}}{C} \cdot\left(l \cdot\left(\mathcal{E}_{\lambda}+k \cdot\left(\mathcal{E}_{\lambda}+1\right)\right)+L \cdot(K+1) \cdot\left(\mathcal{E}_{\lambda}+\mathcal{E}_{w}+1\right)\right)- \\
& -\frac{u \cdot C^{\prime}}{C^{2}} \cdot \frac{r_{u}^{\prime} \cdot X^{2}}{r_{R} \cdot E_{R}} \cdot(l \cdot(k+1) \cdot(\Gamma-l)+L \cdot(K+1) \cdot(\Gamma-L))= \\
& =-\frac{X}{r_{R}} \cdot \frac{u^{\prime}}{C} \cdot\left(\Gamma \cdot \mathcal{E}_{\lambda}+L \cdot(K+1) \cdot \mathcal{E}_{w}+\Gamma-l\right)- \\
& -\frac{u \cdot C^{\prime} \cdot X}{C^{2}} \cdot \frac{r_{u}^{\prime} \cdot X}{r_{R} \cdot E_{R}} \cdot(l \cdot(k+1) \cdot(\Gamma-l)+L \cdot(K+1) \cdot(\Gamma-L))= \\
& \left(\text { since } \mathcal{E}_{\lambda}=\frac{L \cdot(K+1)}{\Gamma} \cdot \frac{r_{R}-1}{E_{R}+r_{R}} \cdot \mathcal{E}_{w}-\frac{\Gamma-l}{\Gamma} \cdot E_{R}\right) \\
& =-\frac{X}{r_{R}} \cdot \frac{u^{\prime}}{C} \cdot\left(L \cdot(K+1) \cdot \frac{r_{R}-1}{E_{R}+r_{R}} \cdot \mathcal{E}_{w}-(\Gamma-l) \cdot E_{R}+L \cdot(K+1) \cdot \mathcal{E}_{w}+\Gamma-l\right)- \\
& -\frac{u \cdot C^{\prime} \cdot Q}{\Gamma \cdot C^{2}} \cdot \frac{r_{u}^{\prime} \cdot X}{r_{R} \cdot E_{R}} \cdot(l \cdot(k+1) \cdot(\Gamma-l)+L \cdot(K+1) \cdot(\Gamma-L))= \\
& =-\frac{X}{r_{R}} \cdot \frac{u^{\prime}}{C} \cdot\left(L \cdot(K+1) \cdot\left(\frac{r_{R}-1}{E_{R}+r_{R}}+1\right) \cdot \mathcal{E}_{w}+(\Gamma-l) \cdot\left(1-E_{R}\right)\right)- \\
& -\frac{u \cdot E_{C}}{\Gamma \cdot C} \cdot \frac{r_{u}^{\prime} \cdot X}{r_{R} \cdot E_{R}} \cdot(l \cdot(k+1) \cdot(\Gamma-l)+L \cdot(K+1) \cdot(\Gamma-L))= \\
& \text { (since } E_{C}=E_{R} \text { ) } \\
& =-\frac{X}{r_{R}} \cdot \frac{u^{\prime}}{C} \cdot\left(L \cdot(K+1) \cdot\left(\frac{r_{R}}{E_{R}+r_{R}}+\frac{E_{R}+r_{R}-1}{E_{R}+r_{R}}\right) \cdot \mathcal{E}_{w}+(\Gamma-l) \cdot\left(1-E_{R}\right)\right)- \\
& -\frac{u \cdot r_{u}^{\prime} \cdot X}{\Gamma \cdot C \cdot r_{R}} \cdot(l \cdot(k+1) \cdot(\Gamma-l)+L \cdot(K+1) \cdot(\Gamma-L))= \\
& \left(\text { since } X \cdot u^{\prime}=R=u \cdot E_{u}, \mathcal{E}_{w}=(L-l) \cdot \frac{E_{R}}{\Gamma}, E_{R}+r_{R}-1=\frac{r_{u}^{\prime} \cdot X}{E_{R}}\right) \\
& =-\frac{u \cdot E_{u}}{C \cdot r_{R}} \cdot\left(L \cdot(K+1) \cdot\left(\frac{r_{R}}{E_{R}+r_{R}}+\frac{r_{u}^{\prime} \cdot X}{\left(E_{R}+r_{R}\right) \cdot E_{R}}\right) \cdot(L-l) \cdot \frac{E_{R}}{\Gamma}+(\Gamma-l) \cdot r_{u}\right)- \\
& -\frac{u \cdot r_{u}^{\prime} \cdot X}{\Gamma \cdot C \cdot r_{R}} \cdot(l \cdot(k+1) \cdot(\Gamma-l)+L \cdot(K+1) \cdot(\Gamma-L))= \\
& =-\frac{u \cdot E_{u}}{C \cdot r_{R}} \cdot\left(L \cdot(K+1) \cdot \frac{L-l}{\Gamma} \cdot \frac{r_{R} \cdot E_{R}}{E_{R}+r_{R}}+(\Gamma-l) \cdot r_{u}\right)-\frac{u \cdot E_{u}}{C \cdot r_{R}} \cdot \frac{L \cdot(K+1) \cdot(L-l)}{\Gamma \cdot\left(E_{R}+r_{R}\right)} \cdot r_{u}^{\prime} \cdot X-
\end{aligned}
$$




$$
\begin{gathered}
-\frac{u \cdot r_{u}^{\prime} \cdot X}{\Gamma \cdot C \cdot r_{R}} \cdot(l \cdot(k+1) \cdot(\Gamma-l)+L \cdot(K+1) \cdot(\Gamma-L))= \\
=-\frac{u \cdot E_{u}}{\Gamma \cdot C \cdot r_{R}} \cdot\left(L \cdot(K+1) \cdot(L-l) \cdot \frac{r_{R} \cdot E_{R}}{E_{R}+r_{R}}+\Gamma \cdot(\Gamma-l) \cdot r_{u}\right)- \\
-\left(L \cdot(K+1) \cdot(L-l) \cdot \frac{E_{u}}{E_{R}+r_{R}}+l \cdot(k+1) \cdot(\Gamma-l)+L \cdot(K+1) \cdot(\Gamma-L)\right) \cdot \frac{u \cdot r_{u}^{\prime} \cdot X}{\Gamma \cdot C \cdot r_{R}}= \\
=-\frac{u}{\Gamma \cdot C \cdot r_{R}} \cdot\left(A^{s}+B^{s} \cdot r_{u}^{\prime} \cdot X\right),
\end{gathered}
$$

where

$$
\begin{gathered}
A^{s}=E_{u} \cdot\left(L \cdot(K+1) \cdot(L-l) \cdot \frac{r_{R} \cdot E_{R}}{E_{R}+r_{R}}+\Gamma \cdot(\Gamma-l) \cdot r_{u}\right)(>0), \\
B^{s}=L \cdot(K+1) \cdot(L-l) \cdot \frac{E_{u}}{E_{R}+r_{R}}+(l \cdot(k+1) \cdot(\Gamma-l)+L \cdot(K+1) \cdot(\Gamma-L))(>0) .
\end{gathered}
$$

Hence, if $r_{u}^{\prime} \geq 0$ then $\frac{d W^{s}}{d \tau}<0$.

\subsection{Proof of Lemma 4}

Note that since

$$
Q\left(\tau_{3}\right)=L \cdot X\left(\tau_{3}\right), \quad q\left(\tau_{3}\right)=l \cdot x\left(\tau_{3}\right),
$$

we obtain from FOC and the Free Entry condition,

$$
E_{R}\left(X\left(\tau_{3}\right)\right)=E_{C}\left(Q\left(\tau_{3}\right)\right), \quad E_{R}\left(x\left(\tau_{3}\right)\right)=E_{C}\left(q\left(\tau_{3}\right)\right) .
$$

5.4.1 Case I): total autarky with $y\left(\tau_{3}\right)=0$

In this case, near $\tau=\tau_{3}$ we have

$$
\begin{gathered}
Q=L \cdot X, \\
q=l \cdot x+k \cdot \tau \cdot l \cdot y, \\
\Pi=L \cdot \frac{R(X)}{\Lambda}-w \cdot C(Q), \\
\pi=l \cdot \frac{R(x)}{\lambda}+k \cdot l \cdot \frac{R(y)}{\lambda}-C(q),
\end{gathered}
$$

where the wage $w$ is constant, since big countries do not trade with other countries and are isolated. The equilibrium equations w.r.t. $(X, x, y, \Lambda, \lambda)$ are

$$
\frac{\partial \Pi}{\partial X}=0, \quad \frac{\partial \pi}{\partial x}=0, \quad \frac{\partial \pi}{\partial y}=0, \quad \Pi=0, \quad \pi=0,
$$

i.e.,

$$
\begin{gathered}
\frac{R^{\prime}(X)}{\Lambda}=w \cdot C^{\prime}, \quad \frac{R^{\prime}(x)}{\lambda}=C^{\prime}, \quad \frac{R^{\prime}(y)}{\lambda}=\tau \cdot C^{\prime}, \\
L \cdot \frac{R(X)}{\Lambda}=w \cdot C(Q), \quad l \cdot \frac{R(x)}{\lambda}+k \cdot l \cdot \frac{R(y)}{\lambda}=C(q) .
\end{gathered}
$$


From Lemma 1, setting $\mathcal{E}_{w}=0$, we have

$$
\begin{gathered}
\frac{d X}{d \tau}=\frac{1}{\tau} \cdot \frac{\mathcal{E}_{\Lambda}}{\frac{R^{\prime \prime}(X)}{R^{\prime}(X)}}, \quad \frac{d x}{d \tau}=\frac{1}{\tau} \cdot \frac{\mathcal{E}_{\lambda}}{\frac{R^{\prime \prime}(x)}{R^{\prime}(x)}}, \quad \frac{d y}{d \tau}=\frac{1}{\tau} \cdot \frac{\mathcal{E}_{\lambda}+1}{\frac{R^{\prime \prime}(y)}{R^{\prime}(y)}} \\
\frac{d Q}{d \tau}=L \cdot \frac{d X}{d \tau}, \quad \frac{d q}{d \tau}=l \cdot \frac{d x}{d \tau}+k \cdot l \cdot\left(y+\tau \cdot \frac{d y}{d \tau}\right) \\
L \cdot \frac{R(X)}{\Lambda} \cdot \mathcal{E}_{\Lambda}=0, \quad l \cdot\left(\frac{R(x)}{\lambda}+k \cdot \frac{R(y)}{\lambda}\right) \cdot \mathcal{E}_{\lambda}=0
\end{gathered}
$$

Hence

$$
\begin{gathered}
\mathcal{E}_{\Lambda}=0, \quad \mathcal{E}_{\lambda}=0, \quad \frac{d X}{d \tau}=0, \quad \frac{d x}{d \tau}=0, \\
\frac{d y}{d \tau}=\frac{1}{\tau} \cdot \frac{1}{\frac{R^{\prime \prime}(y)}{R^{\prime}(y)}}(<0), \\
\frac{d Q}{d \tau}=0, \quad \frac{d q}{d \tau}=k \cdot l \cdot \tau \cdot \frac{d y}{d \tau}(<0),
\end{gathered}
$$

For what concerns prices, from Lemma 1 we get

$$
\begin{gathered}
\mathcal{E}_{P^{X}}=\frac{r_{u}(X)}{r_{R}(X)} \cdot\left(\mathcal{E}_{\Lambda}+\mathcal{E}_{w}\right)-\mathcal{E}_{\Lambda}=0, \quad \mathcal{E}_{p^{x}}=\frac{r_{u}(x)}{r_{R}(x)} \cdot \mathcal{E}_{\lambda}-\mathcal{E}_{\lambda}=0, \\
\mathcal{E}_{p^{y}}=\frac{r_{u}(y)}{r_{R}(y)} \cdot\left(\mathcal{E}_{\lambda / \tau}+1\right)-\mathcal{E}_{\lambda / \tau}=\frac{r_{u}(y)}{r_{R}(y)}=\frac{-\frac{u^{\prime \prime}(y)}{u^{\prime}(y)} \cdot y}{-\frac{R^{\prime \prime}(y)}{R^{\prime}(y)} \cdot y}=\frac{\frac{u^{\prime \prime}(y)}{u^{\prime}(y)}}{\frac{u^{\prime \prime}(y) \cdot\left(2-r_{u^{\prime}}(y)\right)}{u^{\prime}(y) \cdot\left(1-r_{u}(y)\right)}}= \\
=\frac{1-r_{u}(y)}{2-r_{u^{\prime}}(y)}=\frac{1-r_{u}(0)}{2-r_{u^{\prime}}(0)}=\frac{1}{2} .
\end{gathered}
$$

Concerning the mass of firms, from labor balance we get

$$
\begin{gathered}
\mathcal{E}_{N}=\mathcal{E}_{\frac{L}{C(Q}}=\mathcal{E}_{L}-\mathcal{E}_{C(Q) / \tau}=-\mathcal{E}_{C(Q)}=-E_{C}(Q) \cdot \mathcal{E}_{Q}=0 \\
\mathcal{E}_{n}=\mathcal{E}_{\frac{l}{C(q)}}=-\mathcal{E}_{C(q)}=-E_{C}(q) \cdot \mathcal{E}_{q}=-E_{C}(q) \cdot \frac{d q}{d \tau} \cdot \frac{\tau}{q}=-E_{C}(q) \cdot k \cdot l \cdot \tau \cdot \frac{d y}{d \tau} \cdot \frac{\tau}{q}=-k \cdot l \cdot \frac{c \cdot \tau^{2}}{C(q)} \cdot \frac{d y}{d \tau}(>0) .
\end{gathered}
$$

Welfare functions are

$$
W^{B}=L^{2} \cdot \frac{u(X)}{C(L \cdot X)}, \quad W^{s}=l^{2} \cdot \frac{u(x)+k \cdot u(y)}{C(l \cdot x+l \cdot k \cdot y)} .
$$

So we can compute

$$
\frac{d W^{B}}{d \tau}=L^{2} \cdot \frac{u^{\prime}(X) \cdot \frac{d X}{d \tau} \cdot C(Q)-u(X) \cdot C^{\prime} \cdot \frac{d Q}{d \tau}}{(C(Q))^{2}}=0
$$

and

$$
\frac{1}{k \cdot l^{2}} \cdot \frac{d W^{s}}{d \tau}=\frac{1}{k} \cdot \frac{\left(u^{\prime}(x) \cdot \frac{d x}{d \tau}+k \cdot u^{\prime}(y) \cdot \frac{d y}{d \tau}\right) \cdot C(q)-(u(x)+k \cdot u(y)) \cdot C^{\prime} \cdot \frac{d q}{d \tau}}{(C(q))^{2}}=
$$




$$
\begin{gathered}
=\frac{1}{k} \cdot \frac{\left(u^{\prime}(x) \cdot \frac{d x}{d \tau}+k \cdot u^{\prime}(y) \cdot \frac{d y}{d \tau}\right) \cdot C(q)-(u(x)+k \cdot u(y)) \cdot C^{\prime} \cdot l \cdot\left(\frac{d x}{d \tau}+k \cdot\left(\tau \cdot \frac{d y}{d \tau}+y\right)\right)}{(C(q))^{2}}= \\
=\frac{u^{\prime}(y) \cdot C(q)-u(x) \cdot C^{\prime}(q) \cdot l \cdot \tau}{(C(q))^{2}} \cdot \frac{d y}{d \tau}=\frac{R^{\prime}(y) \cdot C(q)-u(x) \cdot C^{\prime}(q) \cdot l \cdot \tau}{(C(q))^{2}} \cdot \frac{d y}{d \tau}= \\
=\frac{\tau \cdot R^{\prime}(x) \cdot C(q)-u(x) \cdot C^{\prime}(q) \cdot l \cdot \tau}{(C(q))^{2}} \cdot \frac{d y}{d \tau}=\frac{\tau \cdot u^{\prime}(x) \cdot E_{R}(x) \cdot C(q)-u(x) \cdot C^{\prime}(q) \cdot l \cdot \tau}{(C(q))^{2}} \cdot \frac{d y}{d \tau}= \\
=\tau \cdot \frac{u(x)}{x} \cdot \frac{\frac{u^{\prime}(x) \cdot x}{u(x)} \cdot E_{R}(x)-\frac{C^{\prime}(q) \cdot l \cdot x}{C(q)}}{C(q)} \cdot \frac{d y}{d \tau}=\tau \cdot \frac{u(x)}{x} \cdot \frac{\frac{u^{\prime}(x) \cdot x}{u(x)} \cdot \mathcal{E}_{R}(x)-\frac{C^{\prime}(q) \cdot q}{C(q)}}{C(q)} \cdot \frac{d y}{d \tau}= \\
=\tau \cdot \frac{u(x)}{x} \cdot \frac{E_{u}(x) \cdot E_{R}(x)-E_{C}(q)}{C(q)} \cdot \frac{d y}{d \tau}=\tau \cdot \frac{u(x)}{x} \cdot \frac{E_{u}(x) \cdot E_{R}(x)-E_{R}(x)}{C(q)} \cdot \frac{d y}{d \tau}= \\
=\tau \cdot \frac{u(x)}{x} \cdot E_{R}(x) \cdot \frac{E_{u}(x)-1}{C(q)} \cdot \frac{d y}{d \tau}(>0) .
\end{gathered}
$$

\subsubsection{Case II): total autarky with $Z\left(\tau_{3}\right)=z\left(\tau_{3}\right)=0$}

In this case, near $\tau=\tau_{3}$ we have

$$
\begin{gathered}
Q=L \cdot X+(k+1) \cdot \tau \cdot l \cdot Z, \\
q=l \cdot x+(K+1) \cdot \tau \cdot L \cdot z, \\
\Pi=L \cdot \frac{R(X)}{\Lambda}+(k+1) \cdot l \cdot \frac{R(Z)}{\lambda}-w \cdot C(Q), \\
\pi=l \cdot \frac{R(x)}{\lambda}+(K+1) \cdot L \cdot \frac{R(z)}{\Lambda}-C(q), \\
W^{B}=L \cdot\left(L \cdot \frac{u(X)}{C(Q)}+l \cdot(k+1) \cdot \frac{u(z)}{C(q)}\right), \\
W^{s}=l \cdot\left(l \cdot \frac{u(x)}{C(q)}+L \cdot(K+1) \cdot \frac{u(Z)}{C(Q)}\right) .
\end{gathered}
$$

The equilibrium equations w.r.t. $(X, Z, x, z, \Lambda, \lambda, w)$ are

$$
\begin{gathered}
\frac{\partial \Pi}{\partial X}=0, \quad \frac{\partial \Pi}{\partial Z}=0, \quad \frac{\partial \pi}{\partial x}=0, \quad \frac{\partial \pi}{\partial z}=0, \\
\Pi=0, \quad \pi=0, \quad T B=0,
\end{gathered}
$$

i.e.,

$$
\begin{gathered}
\frac{R^{\prime}(X)}{\Lambda}=w \cdot C^{\prime}, \quad \frac{R^{\prime}(Z)}{\lambda}=\tau \cdot w \cdot C^{\prime}, \quad \frac{R^{\prime}(x)}{\lambda}=C^{\prime}, \quad \frac{R^{\prime}(z)}{\Lambda}=\tau \cdot C^{\prime} \\
L \cdot \frac{R(X)}{\Lambda}+(k+1) \cdot l \cdot \frac{R(Z)}{\lambda}=w \cdot C(Q), \quad l \cdot \frac{R(x)}{\lambda}+(K+1) \cdot L \cdot \frac{R(z)}{\Lambda}=C(q), \\
\frac{R(Z)}{\lambda \cdot C(Q)}-\frac{R(z)}{\Lambda \cdot C(q)}=0 .
\end{gathered}
$$


Using Lemma 1, FOC and linearity of the cost function we obtain:

$$
\begin{aligned}
& \mathcal{E}_{\Lambda}=-\mathcal{E}_{w}, \quad \mathcal{E}_{\lambda}=0, \quad \mathcal{E}_{w}=\frac{\frac{R^{\prime \prime}(Z)}{\lambda \cdot C(q)}-w^{2} \cdot \frac{R^{\prime \prime}(z)}{\Lambda \cdot C(Q)}}{\frac{R^{\prime \prime}(Z)}{\lambda \cdot C(q)}+w^{2} \cdot \frac{R^{\prime \prime}(z)}{\Lambda \cdot C(Q)}} \\
& \frac{d X}{d \tau}=\frac{d x}{d \tau}=0, \\
& \frac{d Z}{d \tau}=\frac{R^{\prime}(Z)}{R^{\prime \prime}(Z)} \cdot \frac{1}{\tau} \cdot\left(1+\mathcal{E}_{w}\right)=\frac{\frac{2}{\tau} \cdot \frac{R^{\prime}(Z)}{\lambda \cdot C(q)}}{\frac{R^{\prime \prime}(Z)}{\lambda \cdot C(q)}+w^{2} \cdot \frac{R^{\prime \prime}(z)}{\Lambda \cdot C(Q)}}(<0), \\
& \frac{d z}{d \tau}=\frac{R^{\prime}(z)}{R^{\prime \prime}(z)} \cdot \frac{1}{\tau} \cdot\left(1-\mathcal{E}_{w}\right)=\frac{\frac{2}{\tau} \cdot w^{2} \cdot \frac{R^{\prime}(z)}{\Lambda \cdot C(Q)}}{\frac{R^{\prime \prime}(Z)}{\lambda \cdot C(q)}+w^{2} \cdot \frac{R^{\prime \prime}(z)}{\Lambda \cdot C(Q)}}= \\
& =\frac{\frac{2}{\tau} \cdot \frac{R^{\prime}(Z)}{\lambda \cdot C(q)}}{\frac{R^{\prime \prime}(Z)}{\lambda \cdot C(q)}+w^{2} \cdot \frac{R^{\prime \prime}(z)}{\Lambda \cdot C(Q)}} \cdot w^{2} \cdot \frac{R^{\prime}(z)}{R^{\prime}(Z)} \cdot \frac{\lambda \cdot C(q)}{\Lambda \cdot C(Q)}= \\
& =\frac{\frac{2}{\tau} \cdot \frac{R^{\prime}(Z)}{\lambda \cdot C(q)}}{\frac{R^{\prime \prime}(Z)}{\lambda \cdot C(q)}+w^{2} \cdot \frac{R^{\prime \prime}(z)}{\Lambda \cdot C(Q)}} \cdot w^{2} \cdot \frac{1}{w} \cdot \frac{\Lambda}{\lambda} \cdot \frac{\lambda \cdot C(q)}{\Lambda \cdot C(Q)}= \\
& =w \cdot \frac{C(q)}{C(Q)} \cdot \frac{d Z}{d \tau}(<0), \\
& \frac{d Q}{d \tau}=l \cdot(k+1) \cdot \tau \cdot \frac{d Z}{d \tau}=2 \cdot l \cdot(k+1) \cdot \frac{\frac{R^{\prime}(Z)}{\lambda \cdot C(q)}}{\frac{R^{\prime \prime}(Z)}{\lambda \cdot C(q)}+w^{2} \cdot \frac{R^{\prime \prime}(z)}{\Lambda \cdot C(Q)}}(<0), \\
& \frac{d q}{d \tau}=L \cdot(K+1) \cdot \tau \cdot \frac{d z}{d \tau}=2 \cdot L \cdot(K+1) \cdot \frac{w^{2} \cdot \frac{R^{\prime}(z)}{\Lambda \cdot C(Q)}}{\frac{R^{\prime \prime}(Z)}{\lambda \cdot C(q)}+w^{2} \cdot \frac{R^{\prime \prime}(z)}{\Lambda \cdot C(Q)}}(<0) .
\end{aligned}
$$

Concerning the prices, from Lemma 1 we get

$$
\begin{gathered}
\mathcal{E}_{P^{X}}=\frac{r_{u}(X)}{r_{R}(X)} \cdot\left(\mathcal{E}_{\Lambda}+\mathcal{E}_{w}\right)-\mathcal{E}_{\Lambda}=\mathcal{E}_{w}=\frac{\frac{R^{\prime \prime}(Z)}{\lambda \cdot C(q)}-w^{2} \cdot \frac{R^{\prime \prime}(z)}{\Lambda \cdot C(Q)}}{\frac{R^{\prime \prime}(Z)}{\lambda \cdot C(q)}+w^{2} \cdot \frac{R^{\prime \prime}(z)}{\Lambda \cdot C(Q)}} \in(-1,1), \\
\mathcal{E}_{P^{Z}}=\frac{r_{u}(Z)}{r_{R}(Z)} \cdot\left(\mathcal{E}_{\lambda}+\mathcal{E}_{w}+1\right)-\mathcal{E}_{\lambda}=\frac{r_{u}(Z)}{r_{R}(Z)} \cdot\left(\mathcal{E}_{w}+1\right)=\frac{1}{2} \cdot\left(\mathcal{E}_{w}+1\right)=
\end{gathered}
$$




$$
\begin{gathered}
=\frac{1}{1+w^{2} \cdot \frac{\lambda \cdot C(q) \cdot R^{\prime \prime}(z)}{\Lambda \cdot C(Q) \cdot R^{\prime \prime}(Z)}} \in(0,1), \\
\mathcal{E}_{p^{x}}=\frac{r_{u}(x)}{r_{R}(x)} \cdot \mathcal{E}_{\lambda}-\mathcal{E}_{\lambda}=0 .
\end{gathered}
$$

From labor balance we get the elasticities concerning the mass of firms:

$$
\begin{aligned}
& \mathcal{E}_{N}=-\mathcal{E}_{C(Q)}=-E_{C}(Q) \cdot \mathcal{E}_{Q}=-E_{C}(Q) \cdot \frac{d Q}{d \tau} \cdot \frac{\tau}{Q}=-E_{C}(Q) \cdot l \cdot(k+1) \cdot \tau \cdot \frac{d Z}{d \tau} \cdot \frac{\tau}{Q}=-l \cdot(k+1) \cdot \frac{c \cdot \tau^{2}}{C(Q)} \cdot \frac{d Z}{d \tau}(>0), \\
& \mathcal{E}_{n}==-\mathcal{E}_{C(q)}=-E_{C}(q) \cdot \mathcal{E}_{q}=-E_{C}(q) \cdot \frac{d q}{d \tau} \cdot \frac{\tau}{q}=-E_{C}(q) \cdot L \cdot(K+1) \cdot \tau \cdot \frac{d z}{d \tau} \cdot \frac{\tau}{q}=-L \cdot(K+1) \cdot \frac{c \cdot \tau^{2}}{C(q)} \cdot \frac{d z}{d \tau}(>0) .
\end{aligned}
$$

Further,

$$
\begin{gathered}
\frac{1}{L} \cdot \frac{d W^{B}}{d \tau}=L \cdot\left(\frac{u^{\prime}(X)}{C(Q)} \cdot \frac{d X}{d \tau}-\frac{u(X)}{(C(Q))^{2}} \cdot C^{\prime}(Q) \cdot \frac{d Q}{d \tau}\right)+l \cdot(k+1) \cdot\left(\frac{u^{\prime}(z)}{C(q)} \cdot \frac{d z}{d \tau}-\frac{u(z)}{(C(q))^{2}} \cdot C^{\prime}(q) \cdot \frac{d q}{d \tau}\right)= \\
=-L \cdot \frac{u(X)}{(C(Q))^{2}} \cdot C^{\prime}(Q) \cdot \frac{d Q}{d \tau}+l \cdot(k+1) \cdot \frac{u^{\prime}(z)}{C(q)} \cdot \frac{d z}{d \tau}= \\
=-L \cdot \frac{u(X)}{(C(Q))^{2}} \cdot C^{\prime}(Q) \cdot l \cdot(k+1) \cdot \tau \cdot \frac{d Z}{d \tau}+l \cdot(k+1) \cdot \frac{u^{\prime}(z)}{C(q)} \cdot \frac{d z}{d \tau}= \\
=l \cdot(k+1) \cdot\left(-L \cdot \frac{u(X)}{(C(Q))^{2}} \cdot C^{\prime}(Q) \cdot \tau \cdot \frac{d Z}{d \tau}+\frac{u^{\prime}(z)}{C(q)} \cdot \frac{d z}{d \tau}\right)= \\
=l \cdot(k+1) \cdot\left(-L \cdot \frac{u(X)}{(C(Q))^{2}} \cdot C^{\prime}(Q) \cdot \tau \cdot \frac{d Z}{d \tau}+\frac{u^{\prime}(z)}{C(q)} \cdot w \cdot \frac{C(q)}{C(Q)} \cdot \frac{d Z}{d \tau}\right)= \\
=l \cdot(k+1) \cdot \frac{w}{C(Q)} \cdot\left(-L \cdot \frac{u(X) \cdot C^{\prime}(Q)}{C(Q)} \cdot \frac{\tau}{w}+u^{\prime}(z)\right) \cdot \frac{d Z}{d \tau}=
\end{gathered}
$$

(using the fact that $R(X) \equiv u(X) \cdot E_{u}(X)$ )

$$
=l \cdot(k+1) \cdot \frac{w}{C(Q)} \cdot\left(-L \cdot \frac{C^{\prime}(Q) \cdot R(X)}{C(Q) \cdot E_{u}(X)} \cdot \frac{\tau}{w}+u^{\prime}(z)\right) \cdot \frac{d Z}{d \tau}=
$$

(due to the zero-profit condition at autarky: $L \cdot R(X)=\Lambda \cdot w \cdot C(Q)$ )

$$
=l \cdot(k+1) \cdot \frac{w}{C(Q)} \cdot\left(-\frac{C^{\prime}(Q)}{E_{u}(X)} \cdot \tau \cdot \Lambda+u^{\prime}(z)\right) \cdot \frac{d Z}{d \tau}=
$$

(because in autarky $\left.R^{\prime}(z)=u^{\prime}(z) \cdot\left(1-r_{u}(z)\right)=u^{\prime}(z) \cdot\left(1-r_{u}(0)\right)=u^{\prime}(z)\right)$

$$
=l \cdot(k+1) \cdot \frac{w}{C(Q)} \cdot\left(-\frac{C^{\prime}(Q)}{E_{u}(X)} \cdot \tau \cdot \Lambda+R^{\prime}(z)\right) \cdot \frac{d Z}{d \tau}=
$$

(due to the linearity of costs: $C^{\prime}(Q)=C^{\prime}(q)$ )

$$
=l \cdot(k+1) \cdot \frac{w}{C(Q)} \cdot\left(-\frac{C^{\prime}(q)}{E_{u}(X)} \cdot \tau \cdot \Lambda+R^{\prime}(z)\right) \cdot \frac{d Z}{d \tau}=
$$


(due to producer's FOC at autarky $C^{\prime}(q) \cdot \tau \cdot \Lambda=R^{\prime}(z)$ )

$$
\begin{gathered}
=l \cdot(k+1) \cdot \frac{w}{C(Q)} \cdot\left(-\frac{R^{\prime}(z)}{E_{u}(X)}+R^{\prime}(z)\right) \cdot \frac{d Z}{d \tau}= \\
=l \cdot(k+1) \cdot \frac{R^{\prime}(z) \cdot w}{C(Q)} \cdot \frac{E_{u}(X)-1}{E_{u}(X)} \cdot \frac{d Z}{d \tau}
\end{gathered}
$$

the latter expression being strictly positive since $E_{u}(X)<1$.

In a similar way,

$$
\begin{gathered}
\frac{1}{l} \cdot \frac{d W^{s}}{d \tau}=l \cdot\left(\frac{u^{\prime}(x)}{C(q)} \cdot \frac{d x}{d \tau}-\frac{u(x)}{(C(q))^{2}} \cdot C^{\prime}(q) \cdot \frac{d q}{d \tau}\right)+ \\
+L \cdot(K+1) \cdot\left(\frac{u^{\prime}(Z)}{C(Q)} \cdot \frac{d Z}{d \tau}-\frac{u(Z)}{(C(Q))^{2}} \cdot C^{\prime}(Q) \cdot \frac{d Q}{d \tau}\right)= \\
=-l \cdot \frac{u(x)}{(C(q))^{2}} \cdot C^{\prime}(q) \cdot \frac{d q}{d \tau}+L \cdot(K+1) \cdot \frac{u^{\prime}(Z)}{C(Q)} \cdot \frac{d Z}{d \tau}= \\
=-l \cdot \frac{u(x)}{(C(q))^{2}} \cdot C^{\prime}(q) \cdot L \cdot(K+1) \cdot \tau \cdot \frac{d z}{d \tau}+L \cdot(K+1) \cdot \frac{u^{\prime}(Z)}{C(Q)} \cdot \frac{d Z}{d \tau}= \\
=L \cdot(K+1) \cdot\left(-l \cdot \frac{u(x)}{(C(q))^{2}} \cdot C^{\prime}(q) \cdot \tau \cdot \frac{d z}{d \tau}+\frac{u^{\prime}(Z)}{C(Q)} \cdot \frac{d Z}{d \tau}\right)= \\
=L \cdot(K+1) \cdot\left(-l \cdot \frac{u(x)}{(C(q))^{2}} \cdot C^{\prime}(q) \cdot \tau \cdot \frac{d z}{d \tau}+\frac{u^{\prime}(Z)}{C(Q)} \cdot \frac{1}{w} \cdot \frac{C(Q)}{C(q)} \cdot \frac{d z}{d \tau}\right)= \\
=L \cdot(K+1) \cdot \frac{1}{C(q)} \cdot\left(-l \cdot u(x) \cdot \frac{C^{\prime}(q)}{C(q)} \cdot \tau+\frac{u^{\prime}(Z)}{w}\right) \cdot \frac{d z}{d \tau}= \\
=L \cdot(K+1) \cdot \frac{1}{C(q)} \cdot\left(-l \cdot \frac{R(x)}{E_{u}(x)} \cdot \frac{C^{\prime}(q)}{C(q)} \cdot \tau+\frac{R^{\prime}(Z)}{w}\right) \cdot \frac{d z}{d \tau}= \\
=L \cdot(K+1) \cdot \frac{1}{C(q)} \cdot\left(-\frac{C^{\prime}(q)}{E_{u}(x)} \cdot \tau \cdot \lambda+\frac{R^{\prime}(Z)}{w}\right) \cdot \frac{d z}{d \tau}= \\
=L \cdot(K+1) \cdot \frac{1}{C(q)} \cdot\left(-\frac{1}{\mathcal{E}_{u}(x)} \cdot \frac{R^{\prime}(Z)}{w}+\frac{R^{\prime}(Z)}{w}\right) \cdot \frac{d z}{d \tau}= \\
=L \cdot(K+1) \cdot \frac{1}{C(q)} \cdot \frac{R^{\prime}(Z)}{w} \cdot \frac{E_{u}(x)-1}{E_{u}(x)} \cdot \frac{d z}{d \tau}(>0) . \\
= \\
=
\end{gathered}
$$

\subsubsection{Case III): total autarky with $Y\left(\tau_{3}\right)=0$}

Similarly to Case I), in this case, in $\tau=\tau_{3}$ we have

$$
\begin{gathered}
Q=L \cdot X+K \cdot \tau \cdot L \cdot Y, \quad q=l \cdot x, \\
\Pi=L \cdot \frac{R(X)}{\Lambda}+K \cdot L \cdot \frac{R(Y)}{\lambda}-w \cdot C(Q), \quad \pi=l \cdot \frac{R(x)}{\lambda}-C(q),
\end{gathered}
$$

where the wage $w$ is constant since big countries can only trade with each other. 
The equilibrium equations w.r.t. $(X, x, y, \Lambda, \lambda)$ are

$$
\frac{\partial \Pi}{\partial X}=0, \quad \frac{\partial \Pi}{\partial Y}=0, \quad \frac{\partial \pi}{\partial x}=0, \quad \Pi=0, \quad \pi=0,
$$

i.e.,

$$
\begin{aligned}
& \frac{R^{\prime}(X)}{\Lambda}=w \cdot C^{\prime}, \quad \frac{R^{\prime}(Y)}{\Lambda}=w \cdot \tau \cdot C^{\prime}, \quad \frac{R^{\prime}(x)}{\lambda}=C^{\prime}, \\
& L \cdot \frac{R(X)}{\Lambda}+K \cdot L \cdot \frac{R(Y)}{\lambda}=w \cdot C(Q), \quad l \cdot \frac{R(x)}{\lambda}=C(q) .
\end{aligned}
$$

Comparative Statics w.r.t. $\tau$ gives us, using Lemma 1 (setting, as in Case I), $\mathcal{E}_{w}=0$ ),

$$
\begin{gathered}
\frac{d X}{d \tau}=\frac{1}{\tau} \cdot \frac{\mathcal{E}_{\Lambda}}{\frac{R^{\prime \prime}(X)}{R^{\prime}(X)}}, \quad \frac{d Y}{d \tau}=\frac{1}{\tau} \cdot \frac{\mathcal{E}_{\Lambda}+1}{\frac{R^{\prime \prime}(Y)}{R^{\prime}(Y)}}, \quad \frac{d x}{d \tau}=\frac{1}{\tau} \cdot \frac{\mathcal{E}_{\lambda}}{\frac{R^{\prime \prime}(x)}{R^{\prime}(x)}}, \\
\frac{d Q}{d \tau}=L \cdot \frac{d X}{d \tau}+K \cdot L \cdot\left(Y+\tau \cdot \frac{d Y}{d \tau}\right), \quad \frac{d q}{d \tau}=l \cdot \frac{d x}{d \tau}, \\
\mathcal{E}_{\Lambda}=0, \quad \mathcal{E}_{\lambda}=0,
\end{gathered}
$$

i.e.,

$$
\begin{gathered}
\mathcal{E}_{\Lambda}=\mathcal{E}_{\lambda}=\frac{d X}{d \tau}=\frac{d x}{d \tau}=\frac{d q}{d \tau}=0, \\
\frac{d Y}{d \tau}=\frac{1}{\tau} \cdot \frac{R^{\prime}(Y)}{R^{\prime \prime}(Y)}(<0), \quad \frac{d Q}{d \tau}=K \cdot L \cdot \tau \cdot \frac{d Y}{d \tau}=K \cdot L \cdot \frac{R^{\prime}(Y)}{R^{\prime \prime}(Y)}(<0) .
\end{gathered}
$$

For what concerns prices, from Lemma 1 we get

$\mathcal{E}_{P^{X}}=\frac{r_{u}(X)}{r_{R}(X)} \cdot\left(\mathcal{E}_{\Lambda}+\mathcal{E}_{w}\right)-\mathcal{E}_{\Lambda}=0, \quad \mathcal{E}_{P^{Y}}=\frac{r_{u}(Y)}{r_{R}(Y)} \cdot\left(\mathcal{E}_{\Lambda}+\mathcal{E}_{w}\right)+1-\mathcal{E}_{\Lambda}=1, \quad \mathcal{E}_{p^{x}}=\frac{r_{u}(x)}{r_{R}(x)} \cdot \mathcal{E}_{\lambda}-\mathcal{E}_{\lambda}=0$.

Concerning the mass of firms, from labor balance we get

$$
\begin{gathered}
\mathcal{E}_{N}=-\mathcal{E}_{C(Q)}=-E_{C}(Q) \cdot \mathcal{E}_{Q}=-E_{C}(Q) \cdot \frac{d Q}{d \tau} \cdot \frac{\tau}{Q}= \\
=-E_{C}(Q) \cdot K \cdot L \cdot \tau \cdot \frac{d Y}{d \tau} \cdot \frac{\tau}{Q}=-K \cdot L \cdot \frac{c \cdot \tau^{2}}{C(Q)} \cdot \frac{d Y}{d \tau}(>0), \\
\mathcal{E}_{n}=-\mathcal{E}_{C(q)}=-E_{C}(q) \cdot \mathcal{E}_{q}=0 .
\end{gathered}
$$

Welfare functions

$$
W^{B}=L^{2} \cdot \frac{u(X)+K \cdot u(Y)}{C(Q)}, \quad W^{s}=l^{2} \cdot \frac{u(x)}{C(q)} .
$$

allow to compute

$$
\begin{gathered}
\frac{1}{L^{2} \cdot K} \cdot \frac{d W^{B}}{d \tau}=\frac{1}{K} \cdot \frac{\left(u^{\prime}(X) \cdot \frac{d X}{d \tau}+K \cdot u^{\prime}(Y) \cdot \frac{d Y}{d \tau}\right) \cdot C(Q)-(u(X)+K \cdot u(Y)) \cdot C^{\prime}(Q) \cdot \frac{d Q}{d \tau}}{(C(Q))^{2}}= \\
=\frac{1}{K} \cdot \frac{\left(u^{\prime}(X) \cdot \frac{d X}{d \tau}+K \cdot u^{\prime}(Y) \cdot \frac{d Y}{d \tau}\right)}{C(Q)}-
\end{gathered}
$$




$$
\begin{gathered}
-\frac{1}{K} \cdot \frac{(u(X)+K \cdot u(Y)) \cdot C^{\prime}(Q) \cdot\left(\frac{d X}{d \tau}+K \cdot L \cdot\left(\tau \cdot \frac{d Y}{d \tau}+Y\right)\right)}{(C(Q))^{2}}= \\
\left(\operatorname{since} \frac{d X}{d \tau}=Y=0\right) \\
=\frac{u^{\prime}(Y) \cdot C(Q)-u(X) \cdot C^{\prime}(Q) \cdot L \cdot \tau}{(C(Q))^{2}} \cdot \frac{d Y}{d \tau}=u(X) \cdot \frac{\frac{u^{\prime}(Y)}{u(X)}-L \cdot \frac{C^{\prime}(Q)}{C(Q)} \cdot \tau}{C(Q)} \cdot \frac{d Y}{d \tau}= \\
=u(X) \cdot \frac{\frac{R^{\prime}(Y)}{u(X)}-L \cdot \frac{C^{\prime}(Q)}{C(Q)} \cdot \tau}{C(Q)} \cdot \frac{d Y}{d \tau}=u(X) \cdot \tau \cdot \frac{\frac{R^{\prime}(X)}{u(X)}-L \cdot \frac{C^{\prime}(Q)}{C(Q)}}{C(Q)} \cdot \frac{d Y}{d \tau}= \\
\frac{u^{\prime}(X)}{u(X)} \cdot E_{R}(X)-L \cdot \frac{C^{\prime}(Q)}{C(Q)} \cdot \frac{d Y}{d \tau}=\frac{u(X)}{X} \cdot \tau \cdot \frac{u^{\prime}(X)}{u(X)} \cdot X \cdot E_{R}(X)-L \cdot X \cdot \frac{C^{\prime}(Q)}{C(Q)} \\
=\frac{u(X)}{C} \cdot \frac{d Y}{d \tau}= \\
=\frac{u(X)}{X} \cdot \tau \cdot \frac{E_{u}(X) \cdot E_{R}(X)-Q \cdot \frac{C^{\prime}(Q)}{C(Q)}}{C(Q)} \cdot \frac{d Y}{d \tau}=\frac{u(X)}{X} \cdot \tau \cdot \frac{E_{u}(X) \cdot E_{R}(X)-E_{C}(Q)}{C(Q)} \cdot \frac{d Y}{d \tau}= \\
=E_{R} \cdot \frac{d Y}{d \tau}=\frac{u(X) \cdot E_{R}(X)}{X} \cdot \tau \cdot \frac{E_{u}(X)-1}{C(Q)} \cdot \frac{d Y}{d \tau}(>0),
\end{gathered}
$$

and

$$
\frac{d W^{s}}{d \tau}=l^{2} \cdot \frac{u^{\prime}(x) \cdot \frac{d x}{d \tau} \cdot C(q)-u(X) \cdot C^{\prime} \cdot \frac{d q}{d \tau}}{(C(q))^{2}}=0 .
$$

\section{References}

[1] Arkolakis C., Costinot A., Rodrguez-Clare A. (2012), "New Trade Models, Same Old Gains?," American Economic Review 102(1), 94-130.

[2] Arkolakis C., Costinot A., Donaldson D., Rodrguez-Clare A. (2012), "The Elusive ProCompetitive Effects of Trade," mimeo.

[3] Bykadorov I., Molchanov P., Kokovin S. (2015), "Elusive Pro-competitive Effects and Harm from Gradual Trade Liberalization, " Preprint 295, Sobolev Institute of Mathematics SB RAS, Novosibirsk, February 2015.

[4] Bykadorov I., Gorn A., Kokovin S., Zhelobodko E. (2015), "Why are losses from trade unlikely?,"Economics Letters 129, 35-38.

[5] Dixit A., Stiglitz J. (1977), "Monopolistic Competition and Optimum Product Diversity," American Economic Review, 67(3), 297-308.

[6] Krugman P.R. (1979) "Increasing returns, monopolistic competition, and international trade," Journal of International Economics, 9, 469-479.

[7] Melitz M., Redding S.J. (2012), "Heterogeneous Firms and Trade," NBER WP 18652.

[8] Zhelobodko E., Kokovin S., Parenti M., Thisse J.-F. (2012), "Monopolistic competition: Beyond the constant elasticity of substitution," Econometrica, 80(6), 2765-2784. 